\title{
MESA 1: DIABETES MELLITUS TIPO 1 Y ENFERMEDAD CARDIOVASCULAR
}

\section{TABLE 1: TYPE 1 DIABETES MELLITUS AND CARDIOVASCULAR DISEASE}

\author{
Coordinadores: Lázaro González ${ }^{1,2}$, Alejandro Chertkoff ${ }^{3,4}$ \\ Secretaria: Mariana Burgos ${ }^{2,5}$ \\ Expertos invitados: Liliana Triffone ${ }^{6}$, Hugo Sanabria ${ }^{7,8}$ \\ Integrantes: Karina Koleff2, ${ }^{2,}$, Laura Roccatagliata ${ }^{2,10}$, Virginia Rama ${ }^{2,11}$, María Laura Pomares ${ }^{2,12}$, \\ Milena Suppo ${ }^{2,13}$, Susana Apoloni' ${ }^{2,14}$, Carolina Muratore ${ }^{2,15}$, Gabriel Rodríguez ${ }^{2,16}$
}

\section{RESUMEN}

La principal causa de muerte en personas con diabetes mellitus tipo 1 (DM1) es de origen cardiovascular (CV). La duración de la DM1 es uno de los predictores más importantes para infarto agudo de miocardio (IAM), junto con el colesterol de lipoproteínas de baja densidad (cLDL) y HbA1c. El desarrollo de DM1 antes de los 10 años de edad se asocia con un riesgo 90 veces mayor de IAM en mujeres. En la DM1 habría una mayor contribución de un estado de inflamación sistémica de bajo de grado. La combinación de electrocardiograma de ejercicio y una técnica de imagen proporciona valor diagnóstico para la detección de isquemia miocárdica y pronóstico. La evaluación del riesgo $\mathrm{CV}$ en el adulto mayor debe ser individualizado y categorizarlo según funcionalidad y comorbilidades a fin de fijar objetivos de control de factores glucémicos y no glucémicos personalizados. Las personas con enfermedad cardíaca conocida o múltiples factores de riesgo cardiovascular (FRCV) deben tener recomendaciones de ejercicio personalizadas; se recomienda el tratamiento intensificado de la glucemia y de los FRCV asociados. En la población pediátrica y adolescentes con DM1 es esencial la detección y tratamiento precoz de los FRCV a fin de prevenir o retrasar el inicio y progresión de eventos $\mathrm{CV}$.

Palabras clave: enfermedad cardiovascular; diabetes mellitus tipo 1; tratamiento; diagnóstico; control glucémico.

Revista de la Sociedad Argentina de Diabetes 2020; Vol. 54 (71-90)

\section{ABSTRACT}

The leading cause of death in type 1 diabetes mellitus (T1DM) is cardiovascular. The duration of diabetes is one of the most important predictors for acute myocardial infarction (AMI), along with low-density lipoprotein cholesterol (cLDL) and HbA1C. Being diagnosed of T1DM before age 10 is been associated with a 90 times higher risk of AMI in women. It has been proposed, that a low-grade systemic inflammation state to be great prompt contributor.

The combination of exercise electrocardiogram and an imaging technique provides diagnostic value for myocardial ischemia detection and future prognosis. Cardiovascular risk assessment in the older adult should be individualized by categorizing it, according to functionality and patient's co morbidity and customized glycaemic and non-glycaemic targets.

People with known heart disease or multiple cardiovascular risk factors should have personalized exercise recommendations, blood glucose intensified treatment and associated risk factors. In paediatric and adolescent population with T1DM, cardiovascular risk factors early screening, recognition and treatment has become essential to prevent or delay the onset and progression of cardiovascular events.

Key words: cardiovascular disease; type 1 diabetes treatment; diagnosis; glycaemic control.

Revista de la Sociedad Argentina de Diabetes 2020; Vol. 54 (71-90)
Médico concurrente, Grupo de Trabajo Nutrición, Hospital J. F. Muñiz, Diabetólogo del Servicio Penitenciario Federal, Médico de Cabecera del Programa de Atención Médica Integral (PAMI), Ciudad Autónoma de Buenos Aires, Argentina

2 Comité de Graduados, Sociedad Argentina de Diabetes (SAD), Ciudad Autónoma de Buenos Aires, Argentina

3 Director Médico, Centro Médico Diabetológico (Cemediab), Médico concurrente del Hospital de Clínicas José de San Martín, Ciudad Autónoma de Buenos Aires, Argentina

4 Comité de Hipertensión Arterial y otros Factores de Riesgo Cardiovascular, Sociedad Argentina de Diabetes (SAD), Ciudad Autónoma de Buenos Aires, Argentina

5 Médica asociada al Servicio de Clínica Médica del Hospital Italiano de Buenos Aires, Ciudad Autónoma de Buenos Aires, Argentina

6 Jefa de la Sección Nutrición y Diabetes Hospital de Niños R. Gutiérrez, Ciudad Autónoma de Buenos Aires, Argentina
Jefe de la Unidad de Diabetes, Departamento de Cardiología, Instituto Cardiovascular de Buenos Aires (ICBA), Ciudad Autónoma de Buenos Aires, Argentina

8 Director del Consejo de Cardiometabolismo, Sociedad Argentina de Cardiología (SAC), Ciudad Autónoma de Buenos Aires, Argentina

9 Diabetóloga, Consultorio Privado, Roque Sáenz Peña, Provincia de Buenos Aires, Argentina

10 Asesora Médica, Laboratorio Eli Lilly, Ciudad Autónoma de Buenos Aires, Argentina

11 Diabetóloga, consultorios externos, Obra Social de los Empleados de Comercio y Actividades Civiles (OSECAC), Poder Judicial y Federación de Asociaciones de Trabajadores de la Sanidad Argentina (FATSA), Ciudad Autónoma de Buenos Aires, Argentina

12 Coordinadora Médica, Unidad Diabetes, Centro Médico CEGYM, provincia de Corrientes, Corrientes, Argentina 
Revista de la Sociedad Argentina de Diabetes Vol. 54 № 2 Suplemento I Jornadas Conjuntas del Comité de Graduados y el Comité de Hipertensión Arterial y otros FRCV. XVIII Jornadas del Comité de Graduados

Mayo-agosto de 2020: 71-90 ISSN 0325-5247 (impresa) ISSN 2346-9420 (en línea)

13 Médica de Planta, Servicio de Diabetología, Hospital Privado de Córdoba, Córdoba, Argentina

14 Médica de Planta, Servicio de Diabetes del Hospital Universitario Austral, Pilar, Provincia de Buenos Aires, Argentina

15 Médica, consultorio privado, Río Gallegos, provincia de Santa Cruz, Argentina

16 Médico Clínico de Planta, Hospital Independencia, provincia de Santiago del Estero, Santiago del Estero, Argentina

Contacto de la autora: Solange Houssay

E-mail: solhoussay@gmail.com

Correspondencia: Billinghurst $21432^{\circ}$ piso depto " $A$ " (C1425DTQ), Ciudad Autónoma de Buenos Aires, Argentina Fecha de trabajo recibido: 09/07/20

Fecha de trabajo aceptado: 09/08/20

Conflictos de interés: los Dres. Lázaro González, Karina Koleff, Virginia Rama, Gabriel Rodríguez, Carolina Murato- re, Milena Suppo, Mariana Burgos y Susana Apoloni no refieren conflictos de interés vinculados con el tema de actualización y estado del arte abordado por la mesa. La Dra. Liliana Trifone recibió honorarios por disertaciones, asesoría e investigación de los laboratorios Novo Nordisk, Eli Lilly, Sanofi Aventis y Medtronic. El Dr. Hugo Sanabria recibió honorarios como disertante de los laboratorios AstraZeneca, Boehringer Ingelheim, Novo Nordisk, Eli Lilly y Servier, por asesoría de AstraZeneca, Boehringer Ingelheim, Eli Lilly y Novo Nordisk, y por investigación de AstraZeneca, Boehringer Ingelheim, Bayer y Sanofi Aventis. El Dr. Alejandro Chertkoff recibió honorarios como disertante de Merck Sharp \& Dohme, Craveri, Baliarda y Servier. La Dra. María Laura Pomares recibió honorarios por disertaciones, asesoría e investigación de los laboratorios Novo Nordisk, AstraZeneca y Medtronic. La Dra. María Laura Roccatagliata es asesora Médica del Laboratorio Eli Lilly.

\section{INTRODUCCIÓN}

La principal causa de muerte en personas con diabetes mellitus tipo 1 (DM1) es de origen cardiovascular. La fisiopatología que subyace a esta asociación aún no es del todo clara y mucha de la información disponible se extrapola de estudios realizados en DM2 o de cohortes de pacientes sin distinción del tipo de diabetes.

Nuestro propósito es presentar una revisión de los principales aspectos de la epidemiología, clínica, diagnóstico y tratamiento de la enfermedad cardiovascular (ECV) en DM1.

\section{Epidemiología de la enfermedad cardiovascular en personas con DM1}

- Incidencia y prevalencia. La mortalidad en personas con DM1 ha disminuido significativamente en los últimos 40 años debido al mejor manejo de factores glucémicos y no glucémicos. Sin embargo, el riesgo de muerte por todas las causas y cardiovascular en DM1 con inadecuado control glucémico es ocho a 10 veces mayor que en la población general. En personas con DM1 y HbA1c $\leq 6,9 \%$ el riesgo persiste dos veces más elevado, por lo que se infiere que participarían otros factores más allá del control metabólico y de la HbA1c. Según datos del United Kingdom General Practice Research Database (GPRD), el primer evento CV en DM1 ocurre en promedio 10 a 15 años antes que en personas sin diabetes. En el Epidemiology of Diabetes Complications (EDC), de Pittsburgh, la incidencia de eventos de enfermedad coronaria (EC) en adultos (28 a 38 años) con DM1 fue 0,98\% por año y mayor al 3\% anual después de los 55 años, lo que la convierte en la principal causa de muerte en esa población. La tasa de prevalencia de ECV varía según la edad, años de evolución desde el diagnóstico, edad al inicio de la enfermedad, sexo y posiblemente según la etnia 1,2,3,4,5,6,7.

- Duración de la DM. La duración de la diabetes es uno de los predictores más importantes para infarto agudo de miocardio (IAM), junto con el colesterol de lipoproteínas de baja densidad (CLDL) y HbA1c. El desarrollo de DM1 antes de los 10 años de edad se asocia con un riesgo 90 veces mayor de IAM en mujeres y 30 veces mayor de ECV hacia los primeros años de su vida adulta generando una pérdida de años de vida de 17,7 y 14,2 en mujeres y hombres con DM1 respectivamente $8,9,10,11$ (Figura 1).

La ECV contribuye en un $25-50 \%$ de todas las muertes en las personas con DM1 de 10 a 19 años de duración, sin embargo supera el $50 \%$ en aquellas con más de 20 años de evolución ${ }^{12}$.

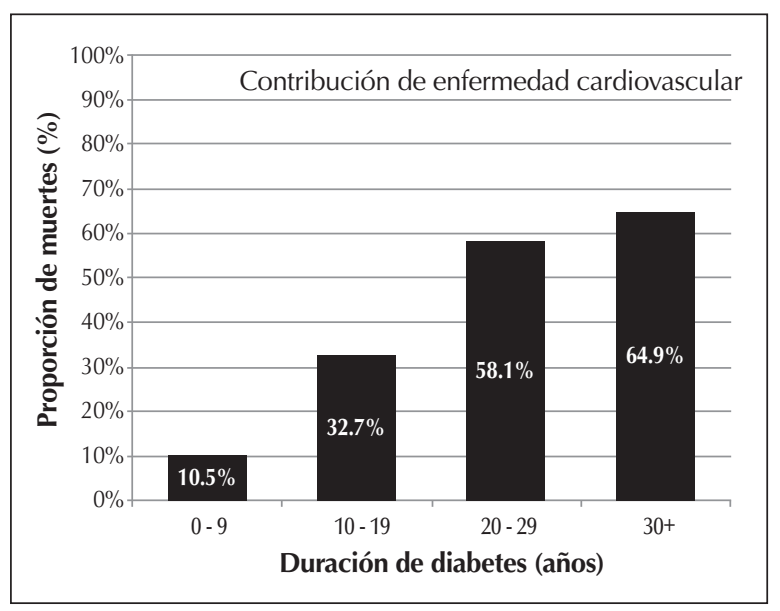

Fuente: Adaptado sin autorización de Secrest AM, et al. ${ }^{6}$.

Figura 1: Proporción de muertes por ECV en cada intervalo de duración de DM. 
- Sexo. En algunos estudios se observó que las mujeres con DM1 tienen hasta un 40\% más de riesgo de mortalidad por todas las causas y el doble de riesgo de eventos cardiovasculares fatales y no fatales en comparación con los hombres. Este exceso de riesgo se debe en parte a que, en la población general, el riesgo de ECV en las mujeres es muy bajo. Ellas presentan mayor deterioro de la sensibilidad a la insulina en la pubertad, peor control glucémico, alteraciones del eje hipotálamo-hipófiso ovárico y mayor proporción de trastornos de la conducta alimentaria. Además, registran una mayor tasa de calcificación coronaria, de disfunción endotelial y lesiones ateroscleróticas más extensas por lo cual se asocia a una pérdida de su cardioprotección premenopáusica 2, 10,13,14.

- Etnia. El estudio New Jersey 725 y el Registro de DM1 infantil del condado de Allegheny sugieren que la ECV es más frecuente en personas de raza negra que en sus pares caucásicos. Sin embargo, el estudio Diabetic Complications Consortium (DiaComp) sugirió tasas similares de ECV en personas con DM1 entre asiáticos, hispanos y en poblaciones no hispánicas, aunque el bajo número de eventos CV no permitió establecer conclusiones ${ }^{15,16,17}$

\section{Diferencia con la población general y con DM2}

La ECV en las personas con DM1 se presenta a edad más temprana y afecta en mayor proporción al sexo femenino. El perfil lipídico aterogénico y la insulinorresistencia asociados al síndrome metabólico (SM) son característicos en la fisiopatología de la DM2. Sin embargo, la epidemia de obesidad en la población general determina la aparición de enfermedad metabólica asociada a las personas con DM1. Por otro lado, existe un aumento de la incidencia de DM2 en niños y adolescentes que dificulta la identificación clínica del tipo de diabetes lo cual determina un peor pronóstico en el manejo de ambas patologías. Como mecanismo diferencial, en DM1 se demostró la presencia de autoinmunidad cardíaca, con positividad de múltiples autoanticuerpos cardíacos. El proceso de aterosclerosis parece ser fisiopatológicamente distinto en DM1, se manifiesta como una enfermedad coronaria de localización más difusa y concéntrica ${ }^{1,2,3,7}$.

Diferentes estudios expusieron la relación entre DM1 y ECV. El más importante fue el Diabetes Control and Complications Trial (DCCT 1983-1993), que incluyó 1.441 pacientes con DM1 con una duración de la enfermedad de 1 a 15 años, divididos en dos ramas: una de tratamiento intensivo y otra convencional. Demostró reducción del $40 \%$ de los ECV en el tratamiento intensivo. El seguimiento de éste, Ilamado Epidemiology of Diabetes Intervention and Complications (EDIC 1993-2012), demostró que los ECV fueron la principal causa de muerte en los pacientes con DM1 (22,4\%), si bien la asociación epidemiológica entre glucemia y enfermedad coronaria (EC) fue débil ${ }^{18}$.

El Pittsburgh Epidemiology of Diabetes Complications Study (EDC), desarrollado entre 1986 y 2012, incluyó 906 pacientes con DM1. Fue un estudio de corte prospectivo con una duración de la enfermedad de 19 años que mostró una tasa de riesgo anual total de EC >al $2 \%$ para individuos con 20 años de diagnóstico. Además determinó que la glucemia predice fuertemente el edema agudo de pulmón (EAP) y la amputación, el accidente cerebrovascular (ACV). La EC tuvo una asociación más baja y el inicio puberal se asoció con mayor riesgo de muerte ${ }^{4}$.

El United Kingdom General Practice Research Database (GPRD), concluido en 2008, incluyó 7.400 pacientes; fue de corte observacional y casos y controles. Concluyó que la ECV afecta igual a ambos sexos por debajo de los 40 años de edad aunque después de la misma a los hombres los afecta más. Los predictores de riesgo fueron nefropatía en hombres y relación cintura cadera e hipertensión arterial (HTA) en mujeres. Por su parte el EURODIAB (Prospective Complication Study), entre 1989 y 1994, con un número de pacientes de 16.362, fue un estudio observacional prospectivo que tuvo una incidencia total de EC (incluyendo cambios en el electrocardiograma) de $9 \%$ en siete años. La hemoglobina glicosilada ( $\mathrm{HbA1c}$ ) no estuvo asociada independientemente con ECV. La proteinuria fue un predictor independiente de EC en ambos sexos y el índice cintura cadera en el sexo masculino $0^{5,19}$.

El Wisconsin Epidemiologic Study of Diabetic Retinopathy (WESDR), de 1982, incluyó pacientes con DM1 y DM2; fue de cohorte prospectivo y tuvo un índice de mortalidad por enfermedad cardíaca isquémica de 9,1 en hombres y 13,5 en mujeres con diagnóstico de DM1 antes de los 30 años, en el cual el predictor mayor fue la nefropatía ${ }^{20}$.

El estudio Coronary Artery Calcification in Type 1 Diabetes (CACTI), de principios de 2000, con 1.416 
pacientes, mostró calcificación arterial coronaria detectable al inicio en el $49 \%$ de los hombres y el $28 \%$ de las mujeres, con progresión durante el seguimiento en $52,5 \%$ de los hombres y $34 \%$ de las mujeres. Esto se asoció con un aumento del riesgo de albuminuria y retinopatía diabética proliferativa ${ }^{21}$.

El estudio de New Jersey, con 725 pacientes, mostró ocho veces más ACV en la etnia blanca, mientras que el Allegheny County Type 1 Diabetes Registry con un N de 1.075 pacientes tuvo una mortalidad por ECV mayor en la etnia blanca. Además asoció el diagnóstico antes de los 10 años de edad con un riesgo 30 veces superior de EC e IAM, y 90 veces superior el riesgo para mujeres y muerte (18 años antes que sus padres sin DM). A su vez, el sexo femenino tuvo $40 \%$ más de riesgo de mortalidad por todas las causas y el doble de riesgo de eventos vasculares fatales, mayor tasa de calcificación de la arteria coronaria, disfunción endotelial y lesiones ateroscleróticas más extensas en comparación con hombres con DM115,16.

El estudio Australian National Diabetes Audit (ANDA), con 1.600 pacientes y 16 años de antigüedad de la enfermedad, demostró que los factores de riesgo cardiovascular más frecuentes fueron HTA (21,9\%), dislipidemia (89,4\%), sobrepeso/ obesidad (56,4\%), tabaquismo (38,5\%), albuminuria $(31,1 \%)$ y $\mathrm{HbA} 1 \mathrm{c} 7 \%(81 \%)^{22}$.

El registro Nacional de Diabetes en Suecia, un estudio de cohorte de 27.195 pacientes, indicó que la edad de inicio de la DM1 es un determinante importante de supervivencia y ECV. El riesgo de muerte prematura por cualquier causa y ECV con mal control glucémico es ocho a 10 veces mayor que en la población general. La HbA1c < a 6,9 tiene un riesgo dos veces más alto. La tasa de amputación no traumática fue del 0,4 al 7,2\% por año. A los 65 años de edad la probabilidad de amputación fue de $11 \%$ en mujeres y $20,7 \%$ en hombres, esto equivale a 86 veces mayor que la población general. El análisis húngaro, con 11.863 pacientes, de cohorte retrospectivo, demostró que la mortalidad por todas las causas fue mayor en pacientes con DM1 con un cociente de riesgo de 2,17 (IC 95\% 1,95-2,41; $P<0,0001$ ). El riesgo de IAM y ACV no fue significativamente diferente en pacientes con DM1 comparado con DM22,23,24.

El Scottish Registry Linkage Study (SRLS) incluyó 21.789 pacientes al comparar individuos con DM1 con personas sin DM. La incidencia del primer ECV y mortalidad en DM1 fue 3 para mujeres y 2,3 para varones, y la mortalidad por todas las causas fue 2,6 en mujeres y 2,7 en varones. La DM1 entre 60 a 69 años se asoció a tres muertes adicionales por cada 100 por año entre los hombres con DM1 (28,51/1.000 personas años en riesgo), y dos por cada 100 por año para mujeres ${ }^{25}$.

El Swedish National Diabetes Register fue un estudio sueco que comparó la terapia con microinfusor de insulina vs las inyecciones múltiples y la mortalidad cardiovascular con 18.168 casos, de los cuales 2.441 usaban infusor y 15.727 inyecciones. Concluyó que el cociente de riesgo para personas con infusores fue menor $(0,55)$ para EC fatal, 0,58 $(0,40$ a 0,85$)$ para ECV fatal (EC y ACV) y $0,73(0,58$ a 0,92$)$ para todas las causas de mortalidad $^{26}$.

\section{Fisiopatología de DM1 y ECV}

La aterosclerosis es un proceso inflamatorio crónico y progresivo. Particularmente en la DM1 habría una mayor contribución de un estado de inflamación sistémica de bajo de grado. Varios estudios demostraron que luego del diagnóstico existiría un aumento de los niveles de marcadores inflamatorios como la proteína $C$ reactiva $(P C R), I L$, fibrinógeno y otros mediadores como el ligando $\mathrm{CD} 40^{7,27,28}$.

La aterosclerosis comienza con la activación y lesión endotelial que lleva al depósito lipídico de LDL modificadas en la íntima y su posterior oxidación para iniciar la formación de la estría grasa y progresar en el desarrollo de la placa aterosclerótica ${ }^{29}$. (Figura 2).

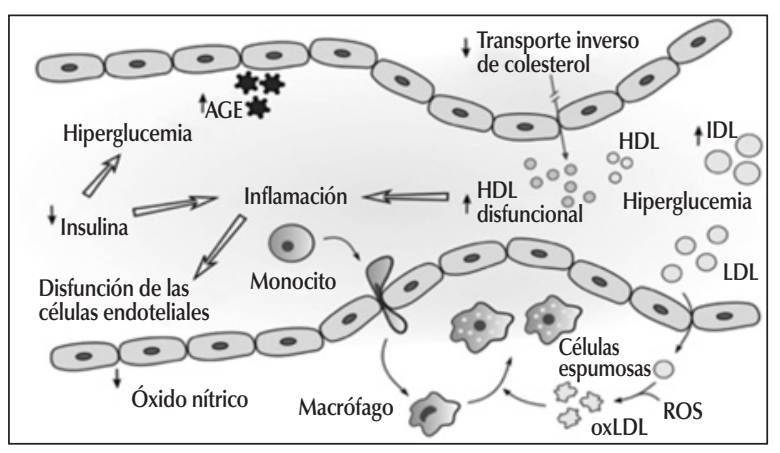

Fuente: Modificado sin autorización de Schofield J, et al. AGE: advanced glycation end; IDL: intermediate density lipoproteins; oxLDL: oxidized low-density lipoprotein (lipoproteína de baja densidad oxidada); ROS: reactive oxygen species (especies reactivas de oxígeno).

Figura 2: Resumen de los mecanismos asociados a ECV en DM1.

El daño vascular inducido por la hiperglucemia crónica se centra en los mecanismos asociados a las complicaciones crónicas de la diabetes, en 
especial la formación de productos de glicación avanzada (en inglés, advanced glycation end products, AGEs) y el aumento en la expresión de sus receptores asociados a ligandos activadores (RAGEs, sus siglas en inglés) ${ }^{30}$. La unión a RAGEs induce la producción de especies reactivas de oxígeno (ROS, sus siglas en inglés) que activan vías proinflamatorias (NFKB), estimulan la proliferación de células musculares lisas y modifican proteínas vasculares. Además, la hiperglucemia y el aumento de ácidos grasos libres generan menor actividad de óxido nítrico endotelial (eNOS) con disminución de óxido nítrico (ON), mayor producción de sustancias vasoactivas como la endotelina-1, la expresión de moléculas de proliferación y adhesión celular (ICAM-1, VCAM-1) $)^{30,31}$.

Se ha demostrado que el polimorfismo genético podría influir en la progresión y el pronóstico de la ECV en personas con DM1. El modelo más estudiado es el genotipo de la haptoglobina que posee tres subtipos. Se halló que el genotipo de haptoglobina 2-2 está presente en un $47 \%$ de las personas con DM1 y se asocia al doble de riesgo de incidencia de ECV comparada con aquellos con el subtipo 1-1. A su vez, hay estudios que describen cómo cambios epigenéticos impulsan la expresión persistente de genes proinflamatorios, incluso después de normalizada la glucemia ("memoria metabólica") 32,33.

\section{Evaluación del riesgo cardiovascular en personas con DM1}

\section{Factores de riesgo tradicionales}

Los factores de riesgo tradicionales parecen funcionar de manera diferente en DM1 determinando un riesgo adicional sinérgico más que aditivo. La edad, la duración de la diabetes, la presión arterial sistólica (PAS), la HbA1c, el cLDL, la albuminuria y la velocidad de filtrado glomerular estimada (VFGe) son variables que interactúan entre sí como factores de riesgo cardiovascular en DM1. La HbA1c y la albuminuria son los predictores más importantes de mortalidad y ECV. La duración de la diabetes, la albuminuria y la PAS tienen interacciones estadísticamente significativas con $\mathrm{HbA} 1 \mathrm{c}^{9,10,11}$.

1) Control glucémico. Nuevas evidencias desde el estudio Diabetes Control and Complications Trial/Epidemiolgy of Diabetes Intervention and Complications (DCCT/EDIC) establecen que el con- trol glucémico óptimo temprano en DM1 lograría a largo plazo beneficios en la reducción de la ECV ${ }^{18}$.

- Hiperglucemia. La hiperglucemia tiene un efecto más marcado en DM1 que en DM2. La $\mathrm{HbA} 1 \mathrm{c}$ se relaciona más fuertemente con eventos cardiovasculares fatales que no fatales, aún con $\mathrm{HbA} 1 \mathrm{c} \leq 6,9 \%$. Existe un $2 \%$ de aumento de RR de mortalidad cardiovascular para cada aumento de $\mathrm{mmol} / \mathrm{mol}$ de HbA1c (HR=1.02; IC95\%, 1,017$1,023)$ o $\sim 23 \%$ por cada $1 \%$ de diferencia de $\mathrm{HbA} 1 \mathrm{c}^{9,10,11 .}$

- Hipoglucemia. En personas con ECV preestablecida, la hipoglucemia se asocia a un HR de 1.61 (IC95\%; 1,17-2,22) para eventos CV. Un estudio de cohorte retrospectivo reciente sobre la base de datos del Clinical Practice Research Datalink (CPRD) informó un HR de mortalidad por todas las causas de 1,98 en pacientes con DM1 que habían experimentado al menos un episodio de hipoglucemia severa ${ }^{10,34,35,36}$.

- Variabilidad glucémica. El incremento de la variabilidad glucémica en adolescentes con DM1 se asocia con un aumento de inflamación. Variaciones en la glucemia, en lugar de valores absolutos o promedios en el tiempo, pueden vincularse más fuertemente con resultados cardiovasculares desfavorables. Se necesitan más estudios longitudinales para explorar los efectos a largo plazo ${ }^{36}$.

\section{2) Hipertensión arterial}

La PAS es uno de los cinco predictores más importantes de mortalidad y ECV en personas con DM1, y presenta una relación casi lineal con la muerte y la insuficiencia cardíaca (IC). La PAS es un poderoso predictor de ECV, particularmente de accidente cerebrovascular (ACV). Por cada aumento de un $\mathrm{mmHg}$ en la PAS se asocia con un riesgo de 1,5\% (HR 1.015; IC95\%, 1,011-1,02) para ACV, lo que representa un riesgo de 16\% (12-20\%) por cada aumento de $10 \mathrm{mmHg}^{8}$.

El estudio Pittsburgh mostró que la HTA fue el principal factor de riesgo de ECV e insuficientemente controlado, atribuyéndose que su manejo subóptimo podría ser causa de la pérdida de protección de ECV asociada al sexo femenino en $\mathrm{DM} 1^{37}$.

\section{3) Dislipemia}

Los niveles plasmáticos de c-LDL se asocian con un riesgo 35-50\% mayor de ECV por cada aumento de un $\mathrm{mmol} / \mathrm{l}(38,7 \mathrm{mg} / \mathrm{dl})$, especialmente asociado a infarto agudo de miocardio (IAM) (HR=1.47; IC95\%, 1,39-1,55). No obstante, el c-LDL no pare- 
ce ser un buen marcador de RCV en la prevención primaria en pacientes con DM1. Los niveles plasmáticos de colesterol aparentemente normales encontrados en personas con DM1 ocultan un perfil lipídico aterogénico, caracterizado por un aumento de lipoproteína de densidad intermedia, LDL pequeñas y densas (LDLpd) y HDL disfuncional. La presencia de hipertrigliceridemia también se asocia a un riesgo de ECV más fuerte que en la población general ${ }^{8,10}$.

\section{4) Enfermedad renal}

La nefropatía diabética aumenta el riesgo de ECV y contribuye al exceso de mortalidad, especialmente después de los primeros 10 años del inicio de la DM1. La albuminuria se asocia con un riesgo entre dos a cuatro veces mayor de complicaciones cardiovasculares y muerte. La VFGe es uno de los predictores más importantes de hospitalización por IC junto con HbA1c, c-LDL, PAS y duración de la diabetes ${ }^{9,38}$.

\section{5) Obesidad e insulinorresistencia}

La obesidad en DM1 se ha asociado a la insulinoterapia intensificada y a la epidemia de obesidad en la población general. Datos del EDIC muestran un incremento de la prevalencia de obesidad del $1-31 \%$ a 12 años de seguimiento. En el EDC, el índice de masa corporal (IMC) asociado con menor mortalidad fue entre $25-30 \mathrm{~kg} / \mathrm{m}^{2}$. La obesidad central como parte del SM implica un incremento exponencial del riesgo CV. El SM aumenta en presencia de nefropatía diabética y se asocia a un control glucémico subóptimo. Aquellos con SM tienen un OR 3,75 veces de presentar nefropatía diabética (IC del 95\%: 2,89-4,85). El estudio Coronary Artery Calcification in Type 1 Diabetes (CACTI) mostró que en DM1 con insulinorresistencia medida por clamp en DM1 era un predictor de la extensión de la calcificación en las arterias coronarias $4,7,21,39$.

\section{6) Tabaquismo}

La prevalencia de tabaquismo en adultos con DM1 en diferentes estudios oscila entre 7-35\%. El consumo de tabaco incrementa el riesgo de nefropatía, retinopatía y neuropatía, posiblemente secundario a un aumento de la inflamación y disfunción endotelial ${ }^{7,40}$.

\section{7) Retinopatía diabética}

Predictor independiente de ECV y mortalidad por todas las causas en DM1 con un $\mathrm{OR}=4.10$ (IC95\%, 1,50-11,18). Las formas graves de reti- nopatía diabética (RD) se asocian con el score de calcificación arterial coronaria (CAC), incluso en ausencia de síntomas ${ }^{41}$.

\section{8) Disautonomía cardíaca}

La disautonomía cardíaca podría precipitar ECV a través de la pérdida de la integridad de las fibras nerviosas simpáticas en arterias epicárdicas por neuropatía autonómica cardíaca (NAC) que contribuiría a un deterioro de la regulación del flujo sanguíneo miocárdico ${ }^{42}$.

\section{Factores de riesgo no tradicionales (emergentes)}

Se han propuesto nuevos marcadores para mejorar la predicción del riesgo CV, sin embargo, la evidencia disponible es escasa como para incluirlos en forma rutinaria.

La Apo B y apolipoproteína CIII se asociaron a ateroesclerosis y nefropatía en DM1. Existiría un menor efecto antinflamatorio ante la presencia de HDL disfuncional. La lipoproteína a (Lpa) es un factor de riesgo independiente y genéticamente determinado para ECV. Estudios observacionales sugieren que los niveles de Lpa mayor de $30 \mathrm{mg} / \mathrm{dl}$ pueden ser de valor en la predicción de ECV en personas con $\mathrm{DM} 1^{10}$.

Elevadas concentraciones plasmáticas de proteína c reactiva (PCR) se correlacionan con marcadores de disfunción endotelial en DM1, incluso en ausencia de ECV. Otros marcadores propuestos son los niveles de cistatina C y MMP-2 ${ }^{10,43}$.

\section{Cálculo del riesgo de ECV en DM1}

Existen distintas calculadoras de riesgo cardiovascular en diabetes, pero ninguna de ellas se adecúa a la población Argentina. La calculadora de riesgo del United Kingdom Prospective Diabetes Study (UKPDS) está diseñada para personas con DM2 y subestima el riesgo de ECV en DM1. La calculadora de riesgo QRISK3-2018 considera la presencia y tipo de DM para determinar el riesgo de desarrollar IAM o ACV en los próximos 10 años, pero se desarrolló para la población inglesa. La calculadora de riesgo Steno T1 predice un primer evento cardiovascular a cinco y 10 años en pacientes con DM1 e incluye 10 factores de riesgo, pero fue desarrollada y validada en la población danesa ${ }^{10}$.

Según las guías de la Sociedad de Cardiología Europea, el riesgo de ECV en DM se puede clasificar en tres categorías. Las personas con riesgo CV muy alto tienen un riesgo de muerte por ECV a 10 años $>10 \%$ (Tabla 1$)^{11}$. 


\begin{tabular}{|l|l|}
\hline $\begin{array}{l}\text { Riesgo } \\
\text { muy alto }\end{array}$ & $\begin{array}{l}\text { Pacientes con DM y ECV establecida } \\
\text { u otro daño de órgano blanco } \\
\text { o tres o más factores de riesgo mayores } \\
\text { o DM1 de inicio temprano de larga duración ( }>20 \text { años) }\end{array}$ \\
\hline $\begin{array}{l}\text { Riesgo } \\
\text { alto }\end{array}$ & $\begin{array}{l}\text { Pacientes con DM duración }>\text { ó=10 años sin daño } \\
\text { de órgano blanco más cualquier otro factor de } \\
\text { riesgo adicional }\end{array}$ \\
\hline $\begin{array}{l}\text { Riesgo } \\
\text { moderado }\end{array}$ & $\begin{array}{l}\text { Pacientes jóvenes (DM1 }<35 \text { años o DM2 }<50 \text { años) } \\
\text { con DM duración }<10 \text { años, sin otros factores de } \\
\text { riesgo }\end{array}$ \\
\hline
\end{tabular}

Fuente: Tomado y adaptado sin autorización de Cosentino F, et al. ${ }^{11}$ a Proteinuria, insuficiencia renal definida por VFGe $\geq 30 \mathrm{ml} /$ min/1,73m², hipertrofia ventricular izquierda o retinopatía.

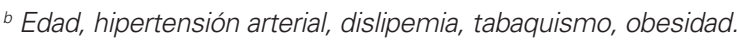

Tabla 1: Categorías de riesgo cardiovascular en DM.

\section{Estudios complementarios para evaluación cardiovascular en DM1}

Si bien en todo paciente con DM1 debe realizarse una evaluación cardiovascular, aún resulta controvertido cómo abordarla11.

El electrocardiograma (ECG) en reposo puede detectar un IAM silente en el $4 \%$ de las personas con DM y se recomienda en todo paciente con DM1. Si bien la medición del grosor de la íntima media carotidea se asocia con ECV, la detección de placas carotideas demostró un valor incremental por encima de dicha medición para detectar ECV asintomática en DM. Además, la placa ecolúcida y el grosor de la placa son predictores independientes de eventos $\mathrm{CV}^{10,11}$.

La ecocardiografía transtorácica, si bien no ha mostrado reducción de eventos, es una he- rramienta de bajo costo y accesible que permite detectar hipertrofia ventricular y anormalidades parietales, así como evaluar función sistólica y diastólica contribuyendo a estratificar estos pacientes. El ecocardiograma, la calcificación arterial coronaria (CAC) y el índice tobillo-brazo (ITB) pueden considerarse como modificadores de riesgo en aquellos con riesgo moderado o alto de ECV. EI ITB se asocia con aumento del riesgo de mortalidad cardiovascular y por cualquier causa en pacientes con y sin $\mathrm{DM}^{11}$.

La combinación de ECG de ejercicio y una técnica de imagen proporciona valor diagnóstico para la detección de isquemia miocárdica y pronóstico incremental en pacientes con DM. La prueba de esfuerzo con imágenes de perfusión miocárdica o ecocardiografía de estrés permite la detección de isquemia miocárdica silente. Las pruebas evocadoras de isquemia pueden indicarse en individuos asintomáticos de muy alto riesgo, con ECG anormal, antecedentes de ACV o enfermedad arterial periférica (EAP), score de calcio coronario elevado, proteinuria o insuficiencia renal ${ }^{11}$.

La evaluación sistemática de presencia de enfermedad coronaria a través de angiotomografía en pacientes con DM1 asintomáticos no demostró mejorar eventos CV mayores comparado con un tratamiento médico agresivo de los factores de riesgo $\mathrm{CV}^{11}$.

La Tabla 2 resume las recomendaciones para evaluar el riesgo CV en pacientes con DM2 que se considera extrapolable a DM1"1․

\begin{tabular}{|c|c|c|}
\hline Recomendaciones & $\begin{array}{l}\text { Clase de } \\
\text { recomendación }\end{array}$ & $\begin{array}{l}\text { Nivel de } \\
\text { evidencia }\end{array}$ \\
\hline $\begin{array}{l}\text { Evaluación rutinaria de albuminuria para identificar pacientes con riesgo de desarrollar disfunción renal o } \\
\text { con alto riesgo de ECV futura }\end{array}$ & I & B \\
\hline Electrocardiograma en pacientes con DM con diagnóstico de HTA o sospecha de ECV & I & C \\
\hline $\begin{array}{l}\text { Considerar evaluación de placas carotideas y/o femorales con ecografía arterial como modificador de riesgo } \\
\text { en pacientes asintomáticos con DM }\end{array}$ & Ila & B \\
\hline $\begin{array}{l}\text { Considerar score CAC por tomografía como modificador en la evaluación de riesgo cardiovascular en } \\
\text { pacientes asintomático con DM con riesgo moderado }\end{array}$ & $\mathrm{IIb}$ & B \\
\hline $\begin{array}{l}\text { Considerar angiografía coronaria por tomografía o imagen funcional (perfusión miocárdica con } \\
\text { radionucleótido, RMN cardíaca, ecocardiografía, estrés con ejercicio o farmacológica) en pacientes } \\
\text { asintomáticos con DM para screening de ECV }\end{array}$ & $\mathrm{IIb}$ & B \\
\hline Considerar ITB como modificador de riesgo en la evaluación del riesgo cardiovascular & $\mathrm{Ilb}$ & B \\
\hline $\begin{array}{l}\text { Considerar detección de placas ateroscleróticas en arterias carótida o femoral por tomografía o RMN como } \\
\text { modificador de riesgo en pacientes con DM con riesgo moderado o elevado de ECV }\end{array}$ & $\mathrm{Ilb}$ & B \\
\hline $\begin{array}{l}\text { No se recomienda ecografía con medición del grosor íntima-media carotídeo como screening de riesgo } \\
\text { cardiovascular }\end{array}$ & III & A \\
\hline No se recomienda la evaluación rutinaria de biomarcadores circulantes para estratificación de riesgo cardiovascular & III & B \\
\hline $\begin{array}{l}\text { No se recomienda el uso de scores de riesgo desarrollados para la población general para la evaluación del } \\
\text { riesgo cardiovascular en pacientes con DM }\end{array}$ & III & $\mathrm{C}$ \\
\hline
\end{tabular}

Fuente: Tomado y adaptado sin autorización de Cosentino F, et al. ${ }^{11}$.

ECV: enfermedad cardiovascular; DM: diabetes mellitus; HTA: hipertensión arterial; CAC: calcificación arterial coronaria;

RMN: resonancia magnética nuclear; ITB: índice tobillo-brazo.

Tabla 2: Recomendaciones para el uso de pruebas de laboratorio, electrocardiograma e imágenes para evaluar el riesgo cardiovascular en pacientes asintomáticos con diabetes. 
Revista de la Sociedad Argentina de Diabetes Vol. 54 № 2 Suplemento I Jornadas Conjuntas del Comité de Graduados y el Comité de Hipertensión Arterial y otros FRCV. XVIII Jornadas del Comité de Graduados

Mayo-agosto de 2020: 71-90 ISSN 0325-5247 (impresa) ISSN 2346-9420 (en línea)

\section{Presentación clínica de ECV en DM1}

\section{Enfermedad coronaria}

- Epidemiología. La DM1 se asocia a un mayor riesgo de EC que la población general y con similar presentación en la DM2. La incidencia de eventos coronarios en pacientes con DM1 es del $16 \%$ a 10 años del diagnóstico y la edad media de inicio de los mismos se encuentra alrededor de los 30 años. La incidencia acumulada de EC oscila entre 2,1 y $19 \%$, y la mayoría de los estudios informa incidencias acumuladas de $15 \%$ durante 15 años de seguimiento ${ }^{7,44}$.

- Fisiopatología. La lesión de la placa aterosclerótica en DM1 se presenta con mayor calcificación de la íntima, rigidez arterial y carga de lípidos y macrófagos cuando se compara con la población control. Estos hallazgos se encuentran desde la primera década del diagnóstico de DM y se observa una estenosis más severa con compromiso más extenso (múltiples vasos) y distal en comparación con la población general ${ }^{7,44,45}$.

La nefropatía diabética es un factor predictivo independiente. El rol del control glucémico es controvertido; no se ha demostrado asociación entre control glucémico/HbA1c y aparición de EC 19,44,46,47.

- Diagnóstico de EC. El diagnóstico de angina crónica estable es clínico a partir del interrogatorio de los síntomas. Una vez establecido, una prueba evocadora de isquemia permitirá una adecuada estratificación. En algunos casos, como pacientes sintomáticos a baja clase funcional, deterioro de la función ventricular o alto riesgo isquémico, se necesitará estudiar la anatomía coronaria por cinecoronariografía (CCG) para evaluar revascularización ${ }^{46,47,48}$.

\section{Enfermedad arterial periférica}

Son predictores para enfermedad arterial periférica (EAP) la edad, sexo masculino, antecedentes de lesiones en los pies o úlceras, HTA, niveles de c-LDL, HbA1c, duración de la DM1, albuminuria, filtrado glomerular disminuido, tabaquismo y retinopatía diabética. En un metaanálisis, que incluyó cinco estudios con pacientes DM1, se observó que con cada aumento del $1 \%$ en $\mathrm{HbA} 1 \mathrm{c}$ el riesgo de EAP aumentó en un $18 \%$. Sin embargo el control glucémico agresivo no redujo la tasa de oclusión arterial pero sí disminuyó la calcificación arterial periférica7,49.

- Epidemiología. En DM1 la tasa de amputación ocurre entre $0,4-7,2 \%$ por año. Datos de una base sueca informó que a los 65 años de edad en personas con DM1, la probabilidad acumulada de amputación fue de 11 y $20 \%$ para mujeres y hombres respectivamente. La tasa de amputación en esta población era casi 86 veces mayor que en la población general?

- Diagnóstico. La EAP puede ser asintomática o presentar síntomas típicos, como la claudicación intermitente, dolor en reposo, ulceración distal o gangrena. El diagnóstico se realiza por anamnesis y examen físico CV. La medición del ITB puede ser de utilidad pero muchas veces la calcificación arterial, comúnmente vista en pacientes con diabetes, puede hacer no compresibles las arterias resultando un ITB erróneo. Por lo tanto, puede ser de mayor utilidad el índice dedo-brazo. El eco-doppler arterial de miembros inferiores puede evaluar la presencia de placas y su repercusión hemodinámica. La evaluación anatómica a través de resonancia, angiotomografía o estudio hemodinámico puede indicarse en aquellos pacientes que se plantea revascularización. Se aconseja el uso combinado de los mismos o la adición de otros métodos como la perfusión transcutánea de oxígeno. La EAP tiene iguales objetivos de prevención secundaria en cuanto al control de los factores de riesgo que la enfermedad cardiovascular ${ }^{49,50,51}$.

\section{Neuropatía autonómica cardíaca}

- Epidemiología. Las estimaciones de la prevalencia de NAC en DM1 varían ampliamente debido a las diferentes definiciones y prueba de diagnóstico utilizadas. Se considera que puede afectar hasta el $40 \%$ de las personas con DM1. La isquemia miocárdica silente es más común en presencia de NAC?

- Diagnóstico. La neuropatía en DM1 puede manifestarse como taquicardia o bradicardia en reposo, intolerancia al ejercicio, hipotensión ortostática, pérdida de la disminución nocturna de la presión arterial o isquemia miocárdica silenciosa. Un indicador temprano es la variabilidad reducida de la frecuencia cardíaca, evaluada clínicamente por una frecuencia cardíaca fija de 80 a 90 latidos por minuto. Para su diagnóstico se utiliza el Test de Ewing?.

\section{Enfermedad cerebrovascular}

- Epidemiología. La DM1 se asocia con incremento de ACV desde edades más tempranas que en la población general. En registros suecos la tasa de incidencia en personas con DM1 de 15 a 49 años es de 130/100.000 personas/año vs controles de $8 / 100.000$ con una tasa de incidencia estandarizada ( $\mathrm{TIE}$ ) de 17,9 y 26,1 en hombres y mujeres 
respectivamente. El riesgo acumulativo a 10 años para ACV isquémico fatal o no fatal en personas con DM1 entre 15 a 49 años es de 40,9\%, y es menor en DM2 $(29,7 \%)$ y en los controles $(12 \%)^{52,53}$.

- Fisiopatología. Se describen los mismos factores de riesgo $\mathrm{CV}$ que en la enfermedad aterosclerótica: disfunción endotelial, inflamación sistémica y rigidez arterial. Existirían cambios tempranos en las carótidas con incremento del grosor de la íntima arterial y aterosclerosis temprana ${ }^{54}$.

- Presentación clínica y factores de riesgo. El $\mathrm{ACV}$ isquémico es la forma de presentación clínica más frecuente como se observa en la Tabla $3^{55}$.

En registros suecos (Swedish National DT Register) de personas con DM1 con una edad media de 35,5 años y 20 años de evolución, luego de un período de seguimiento por ocho años, se encontraron como principales factores de riesgo a la HbA1c, la proteinuria y la dislipemia. No se halló relación con los años de duración de la diabetes. En el mismo estudio se detectó que las personas con DM1 tuvieron un mayor riesgo de ACV isquémico (RR ACV isquémico: 3,29 vs hemorrágico: 2,49 ), excepto en el grupo con mayores niveles de $\mathrm{HbA} 1 \mathrm{C}$, donde fue mayor el riesgo de ACV hemorrágico en aquellos con $\mathrm{HbA} 1 \mathrm{c}>9,7 \%$ (HR ACV isquémico 7,9 y hemorrágico 8,17$)^{56}$.

Mientras que para el ACV isquémico se observó que los principales factores de riesgo fueron la duración de DM1 (RR 1.06 [IC 95\% 1,04-1,08]), la nefropatía diabética $(2,81[1,75-4,71])$, la PAS $(1,02$ $[1,01-1,03])$, el tabaquismo $(1,93[1,23-3,02])$ y la HbA1c (1,23 [1,06-1,41]); en el ACV hemorrágico se han descripto: IMC $(0,89[0,81-0,98])$, nefropatía diabética $(2,77[1,20-6,42])$, PAS $(1,02[1,00-1,03])$ y retinopatía diabética severa $(2,99[1,18-7,55])^{57}$.

\begin{tabular}{|c|c|c|c|c|c|}
\hline & \multicolumn{5}{|c|}{ Odds ratio/riesgo relativo/cociente de riesgo } \\
\hline $\begin{array}{c}\text { ACV } \\
\text { isquémico }\end{array}$ & $\begin{array}{c}\text { ACV } \\
\text { trombótico }\end{array}$ & $\begin{array}{c}\text { Infarto } \\
\text { oclusivo } \\
\text { (grandes } \\
\text { arterias) }\end{array}$ & $\begin{array}{c}\text { Infarto } \\
\text { lacunar } \\
\text { (pequeños } \\
\text { vasos) }\end{array}$ & $\begin{array}{c}\text { Hematoma } \\
\text { intracerebral }\end{array}$ & $\begin{array}{c}\text { ACV } \\
\text { Total }\end{array}$ \\
\hline 6,3 & 6,4 & 7,2 & 7,2 & 3,8 & 4,7 \\
$(4,0-9,80)$ & $(3,9-10,5)$ & $(3,2-16,2)$ & $(3,2-16,1)$ & $(1,2-11,8)$ & $(3,3-6,6)$ \\
\hline
\end{tabular}

Fuente: Adaptado sin autorización de Janghorbani $M$, et al. ${ }^{55}$. ACV: accidente cerebrovascular.

Tabla 3: Presentación clínica.

El ACV hemorrágico tiene menor frecuencia de aparición, pero la sobrevida al año es menor que en el ACV isquémico (52 vs $87 \%$ respectivamente) $)^{58}$.

En la mayoría de los estudios, la PAS se en- cuentra fuertemente asociada con $\mathrm{ACV}$, con un $R R$ de $1.20(1,11-1,29)^{59}$. Otros factores de riesgo son la microalbuminuria, HR: $3.2(1,9-5,6)$, la macroalbuminuria, HR: 4.9 (2.9-8.2), la enfermedad renal terminal, HR: $7.5(4,2-13,3)$ y la retinopatía diabética severa, HR: $3.0(1,9-4,5)^{60}$.

- Estudios diagnósticos. Historia clínica: interrogar sobre la presencia de HTA, retinopatía y nefropatía, factores de riesgo fuertemente asociados a ACV; exámenes de laboratorio; estudios por imágenes (TAC de cerebro para diferenciar isquémico vs hemorrágico; RMN de cerebro) y angiográficos, así como estudios para detectar fuentes embolígenas en casos con sospecha de causa cardioembólica ${ }^{60}$.

\section{Insuficiencia cardíaca}

Se define como la enfermedad del músculo cardíaco donde el miocardio es estructuralmente y funcionalmente anormal, en ausencia de enfermedad arterial coronaria, HTA, enfermedad valvular o desórdenes congénitos. Se presenta inicialmente con hipertrofia cardíaca, fibrosis intersticial y un fenotipo restrictivo con disfunción diastólica, que puede evolucionar a la posterior aparición de IC con sintomatología clínica y disfunción sistólica. Sin embargo, en algunos trabajos se ha descripto que la cardiomiopatía diabética (CMD) podría presentarse de inicio con fenotipo dilatado, con remodelado excéntrico del ventrículo izquierdo (VI), fracción de eyección (FEy) del VI reducida y disfunción sistólica, relacionándose con muerte del cardiomiocito por autoinmunidad ${ }^{61}$.

- Epidemiología. La prevalencia de IC en DM1 es del 19 al 26\%. En un estudio con 20.985 personas con DM1 la incidencia de IC fue 3,38 eventos por 1.000 pacientes/año (IC95\%, 3,12-3,65). El $\mathrm{RR}$ de $\mathrm{IC}$ en personas con DM1 con mal control metabólico ( $\mathrm{HbA} 1 \mathrm{c} \geq 10,5 \%)$ fue de 3,98 (IC95\%, $2,23-7,14)$ comparado con personas con buen control $(\mathrm{HbA} 1 \mathrm{c}<6,5 \%)$. La incidencia fue de 1,42 a 5,20/1.000 personas/año en relación a HbA1c $6,5 \%$ vs $10,5 \%$ respectivamente. En personas con DM1, cada $1 \%$ de incremento de $\mathrm{HbA} 1 \mathrm{c}$ se asoció con un $30 \%$ de aumento de incidencia de $\mathrm{IC}$, independientemente de otros factores de riesgo CV. En personas con DM1 de 55 a 64 años la incidencia fue de 2,1/1.000 personas/año y en cohortes de 41 a 45 años de 2,4/1.000 personas/ año. Los factores de riesgo estadísticamente significativos fueron los mayores niveles de $\mathrm{HbA} 1 \mathrm{c}$ (RR 1.30), IMC (RR 1.05), edad (RR 1.64), duración de la DM (RR 1.34) y PAS (RR 1.15) 
- Fisiopatología. La hiperglucemia crónica determina cambios metabólicos en el cardiomiocito y establece anormalidades morfológicas y funcionales. En algunos estudios se ha demostrado la aparición de cambios funcionales subclínicos del VI como la alteración de la distensión longitudinal del VI en niños con DM1, medidos a través de estudios de imágenes. Dicha alteración podría considerarse como un marcador temprano de CMD subclínica, la cual se caracteriza por anormalidades estructurales y funcionales que incluyen hipertrofia, dilatación, fibrosis y alteración de la señalización celular del cardiomiocito. Los cambios fisiopatológicos que determinan fibrosis y rigidez predisponen más tardíamente a la IC. En ecocardiogramas de personas jóvenes con DM1 de 5,6 años de evolución ya se evidencia la aparición temprana de disfunción diastólica $62,63,65$.

Existen distintos fenotipos de IC en la CMD.

- CMD con fenotipo restrictivo con FEy preservada: fibrosis intersticial por productos de glicación avanzada y lipotoxicidad.
- CMD con fenotipo dilatado con FEy disminuida: muerte del cardiomiocito por autoinmunidad, alteración microvascular e hipoxia ${ }^{63}$.

\section{- Evolución de la cardiomiopatía a IC clíni-} ca. El primer estadio de la CMD es clínicamente asintomático y puede evolucionar a la IC clínica.

- Estadio I: fibrosis y rigidez con disminución de la compliance del VI caracterizada por hipertrofia y deterioro del llenado diastólico, incremento del llenado atrial y prolongación de la relajación isovolumétrica con aumento de la presión de fin de diástole del VI.

- Estadio II: hipertrofia del VI, remodelado cardíaco, disfunción diastólica del VI y aparición de síntomas de IC con FEy conservada (FEy $>50 \%$ ).

- Estadio III: dilatación del VI, disfunción sistólica con disminución de la FEy (FEy $<50 \%$ ) y aparición de IC clínica (disnea, ritmo de galope, signos de congestión pulmonar).

La CMD puede clasificarse según se observa en la Tabla $4^{64}$.

\begin{tabular}{|l|l|l|l|l|}
\hline Estadio CMD & Estadio 1 & Estadio 2 & Estadio 3 \\
\hline & $\begin{array}{l}\text { IC diastólica con fracción } \\
\text { de eyección preservada }\end{array}$ & $\begin{array}{l}\text { IC sintomática con } \\
\text { disfunción sistólica y } \\
\text { diastólica }\end{array}$ & $\begin{array}{l}\text { IC sintomática con } \\
\text { contribución de } \\
\text { HTA, enfermedad } \\
\text { microvascular o viral }\end{array}$ & $\begin{array}{l}\text { IC sintomática con } \\
\text { contribución de } \\
\text { enfermedad arterial } \\
\text { coronaria }\end{array}$ \\
\hline $\begin{array}{l}\text { American Colleage of } \\
\text { Heardiology/ American } \\
\text { ACC/AHA) }\end{array}$ & $\begin{array}{l}\text { Persona con riesgo } \\
\text { asintomática y sin } \\
\text { cambios estructurales }\end{array}$ & $\begin{array}{l}\text { Asintomática con } \\
\text { cambios estructurales } \\
\text { cardíacos }\end{array}$ & $\begin{array}{l}\text { Estadio C sintomática, con } \\
\text { cambios estructurales } \\
\text { cardíacos }\end{array}$ \\
\hline Clase funcional & Clase 1 & $\begin{array}{l}\text { IC refractaria que requiere } \\
\text { intervenciones especiales }\end{array}$ \\
\hline $\begin{array}{l}\text { New York Heart } \\
\text { Association (NYHA) }\end{array}$ & $\begin{array}{l}\text { Asintomática, sin } \\
\text { limitación de la actividad }\end{array}$ & $\begin{array}{l}\text { Leve limitación durante la } \\
\text { actividad física habitual. } \\
\text { Fatiga, palpitaciones, } \\
\text { disnea o angina }\end{array}$ & $\begin{array}{l}\text { Marcada limitación, } \\
\text { con síntomas durante } \\
\text { actividad física mínima }\end{array}$ & $\begin{array}{l}\text { Presencia de síntomas en } \\
\text { reposo. No puede realizar } \\
\text { actividades diarias sin } \\
\text { disconfort }\end{array}$ \\
\hline
\end{tabular}

Fuente: Gulsin GS, et al. ${ }^{64}$.

IC: insuficiencia cardíaca; HTA: hipertensión arterial; CMD: cardiomiopatía diabética.

Tabla 4: Clasificación de la CMD.

- Métodos diagnósticos. Los más frecuentemente utilizados son: ecocardiograma doppler color, tomografía axial computarizada (TAC) y RNM cardíaca, los cuales permiten detectar cambios en la estructura (fibrosis) y la función cardíaca ${ }^{61}$.

- IC diastólica (fenotipo restrictivo): ecocardiograma doppler. FEy $>50 \%$, índice de llenado de fin de diástole del VI $<97 \mathrm{ml} / \mathrm{m}^{2}$. Doppler tisular $E^{\prime} E>15$.

- IC sistólica (fenotipo dilatado): ecocardiograma doppler. FEy $<50 \%$, índice de llenado de fin de diástole del $\mathrm{VI}>97 \mathrm{ml} / \mathrm{m}^{2}$.

Es importante aclarar que en todo paciente con DM1 e IC deberá descartarse la cardiopatía isquémica a través de estudios funcionales o anatomía coronaria según su valoración individual.

- Biomarcadores. Niveles elevados de péptido natriurético atrial y péptido natriurético cerebral (brain natriuretic peptide, BNP) pueden servir como marcadores tempranos en los primeros estadios de CMD e IC. Sin embargo, el BNP puede tener falsos positivos en pacientes con obesidad, mayor edad, uso de inhibidores del sistema renina angiotensina y disfunción renal. Por el momento estudios de imágenes, laboratorios y ergometría no son lo suficientemente sensibles para diagnos- 
ticar la disfunción subclínica en los primeros estadios de $\mathrm{CMD}^{64}$.

\section{Intervenciones no farmacológicas en personas con DM1 y ECV}

Existe escasa literatura sobre estrategias no farmacológicas efectivas para el manejo de ECV en DM1, por lo cual las recomendaciones en esta población se basan en la evidencia obtenida de estudios en DM2.

Las guías incluyen recomendaciones sobre estilo de vida saludable que consensuan objetivos nutricionales con intervenciones activas para el manejo de la obesidad abdominal, disminuyen el consumo de sodio, evitan el sedentarismo, establecen metas de actividad física alcanzables, efectivizan el asesoramiento sobre el cese tabáquico y el consumo moderado de alcohol, e incluyen a los pacientes en programas educativos locales estructurados a fin de optimizar el control glucémico y otros factores de riesgo CV (FRCV).

- Educación. Es esencial promover la autogestión en diabetes para fomentar la adherencia al tratamiento. La información debe incluir consejos sobre estilo de vida saludable, monitoreo glucémico, administración apropiada de fármacos, manejo en días de enfermedad, ventajas y efectos adversos potenciales de los medicamentos prescriptos ${ }^{65,66}$.

- Peso saludable. Los estudios DCCT/EDIC, EDC, CACTI y EURODIAB mostraron aumento en la prevalencia de la obesidad en DM1 y SM, y detectaron un efecto sinérgico sobre ECV por su asociación con insulinorresistencia. La obesidad, en especial la distribución central, se asocia a mayor adiposidad visceral, HTA, dislipemia y resistencia a la insulina en DM1. Además, es un factor de riesgo de ECV que se ha relacionado con aumento de la morbimortalidad ${ }^{4,19,21}$.

- Asesoramiento nutricional. No hay diferencias respecto de la alimentación sugerida en DM2, pero se refuerza el conteo de hidratos de carbono con un enfoque individualizado y flexible según el esquema de insulinoterapia utilizado. Se sugiere sustituir grasas saturadas por grasas monoinsaturadas y poliinsaturadas (similar a la dieta mediterránea) por su asociación con menor riesgo de aterosclerosis; incentivar el consumo de alimentos ricos en ácidos grasos omegas n-3 de cadena larga, presente en el pescado (EPA y DHA), las nueces y semillas; se recomienda evitar alimentos y bebidas con un índice glucémico alto y aumentar el consumo de fibra soluble a través de la ingesta de frutas y verduras (fuentes de potasio y magnesio). También debe desalentarse el consumo excesivo de alcohol dado que inhibe la gluconeogénesis, favorece la hipoglucemia y promueve la pérdida de autocuidado. El consumo de sodio sugerido es $<2.300 \mathrm{mg} / \mathrm{día}^{67,68}$.

- Inactividad física y sedentarismo. El ejercicio combinado aeróbico y de resistencia mejora la presión arterial, el control glucémico, la sensibilidad a la insulina, la función endotelial, la fuerza muscular, los niveles de c-LDL y las complicaciones microvasculares. El incremento del fitness cardiorrespiratorio mejora los parámetros metabólicos y reduce la morbimortalidad CV. La actividad física estructurada también parece mejorar la enfermedad macrovascular en personas con DM1. Las principales barreras son la hipoglucemia y el control glucémico subóptimo $69,70,71$.

Las personas con enfermedad cardíaca conocida o múltiples factores de riesgo cardiovascular deben tener recomendaciones de ejercicio personalizadas. Se recomienda un mínimo de 120-150 minutos de actividad aeróbica moderada por semana, combinada con al menos dos sesiones de entrenamiento de fuerza por semana ${ }^{69}$.

- Tabaco. Su consumo aumenta la morbimortalidad por su asociación a enfermedad micro y macrovascular mediante mecanismos como el estrés oxidativo y la disfunción endotelial. Es un predictor de mal control metabólico. La consejería para cese tabáquico debe realizarse en forma sistemática ${ }^{72}$.

\section{Otros factores considerados}

- Depresión. Si bien es más frecuente en personas con DM1 que en aquellas con DM2, es muy baja su pesquisa diagnóstica. La depresión se considera un factor de riesgo independiente significativo para la ECV y empeora su pronóstico en personas con $\mathrm{DM}^{73}$.

- Apnea obstructiva del sueño. Se asocia con mayor riesgo de morbimortalidad por ECV y debe considerarse, identificarse y tratarse adecuadamente ${ }^{74}$.

- Periodontitis. El examen odontológico debe considerarse como parte de la evaluación y protección del riesgo de ECV75.

- Situación socioeconómica. El nivel socioeconómico bajo en poblaciones de mayor edad que están aisladas y particularmente tienen diabetes aumenta el riesgo de muerte por $\mathrm{ECV}^{76}$. 


\section{Intervenciones farmacológicas en personas adultas con DM1 y ECV}

Las guías actuales de manejo de ECV en DM1 se extrapolan de investigaciones realizadas en población con DM2 ya que no hay estudios con relevancia estadística disponibles en DM1 y menos aún en menores de 30 años, adolescentes y niños. La mayoría de las recomendaciones se basa en consensos de expertos y en estudios de cohorte. El tratamiento agresivo de los múltiples factores de riesgo cardiovascular es la clave para mejorar los resultados a largo plazo69,77,78,79.

- Hipertensión arterial. Según las Guías Internacionales y Recomendaciones Nacionales del Consenso Argentino de HTA 2018, el manejo es similar a las personas con DM2 para buscar un objetivo general de TA $<140 / 90 \mathrm{mmHg}$. En la Figura 3 se describen opciones terapéuticas y seguimiento ${ }^{80}$.

- Dislipemia. Considerando la Guía de Práctica Clínica de la Sociedad Argentina de Lípidos 2019 y las Guías Internacionales, se propone un abordaje similar a personas con DM2. En aquellas menores de 40 años, donde las evidencias son escasas, se plantea el uso de estatinas ante la presencia de daño de órgano blanco o FRCV asociados según el LDL basal. En mayores de 40 años la mayoría de los pacientes con DM1 requerirá estatinas. Por otro lado, la European Society of Cardiology (ESC) y la European Association for the Study of Diabetes (EASD) establecen objetivos de control más estrictos (Tabla 5) ${ }^{11,81}$.

\begin{tabular}{|l|l|l|}
\hline Categoría & Definición & Objetivos \\
\hline $\begin{array}{l}\text { Muy alto } \\
\text { riesgo }\end{array}$ & $\begin{array}{l}\text { ECV establecida o daño de órgano } \\
\text { blanco (proteinuria, disfunción } \\
\text { renal, HVl, retinopatía) o }>/ 3 \mathrm{FR} \text { o } \\
\mathrm{DM} 1>/ 20 \text { años }\end{array}$ & $\mathrm{LDL}<55 \mathrm{mg} / \mathrm{dl}$ \\
\hline Alto riesgo & $\begin{array}{l}\text { Duración DM }>10 \text { años y } 1 \mathrm{FR} \\
\text { sin daño de órgano blanco }\end{array}$ & $\mathrm{LDL}<70 \mathrm{mg} / \mathrm{dl}$ \\
\hline $\begin{array}{l}\text { Moderado } \\
\text { riesgo }\end{array}$ & $\begin{array}{l}\text { Pacientes } \mathrm{DM} 1<35 \text { años con } \\
\text { duración } \mathrm{DM}<10 \text { años sin } \\
\text { otros FR }\end{array}$ & $\mathrm{LDL}<100 \mathrm{mg} / \mathrm{dl}$ \\
\hline
\end{tabular}

Fuente: Cosentino et al. ${ }^{11}$.

ECV: enfermedad cardiovascular; FR: factor de riesgo.

Tabla 5: Objetivos de LDL.

\section{- Control glucémico}

- Insulina: el DCCT/EDIC demostró que el control glucémico intensivo redujo la tasa de eventos CV a través de la disminución en la calcificación de arterias coronarias y la progresión de la aterosclerosis, aunque todavía faltan datos para avalar que dicho control tiene efecto en parámetros estructurales y funcionales del miocardio y el sistema vascular. Sin embargo, se recomienda el tratamiento intensificado de la glucemia y de los FRCV asociados. Además se ha observado que la dosis de insulina no aumenta el riesgo de evento CV per $s e^{82,83,84}$.

- Metformina: su uso no ha demostrado en DM1 que reduzca las complicaciones crónicas ni la mortalidad. Sólo se evidenció reducción de la dosis diaria total de insulina y del perfil lipídico, aunque tampoco se sostuvo su efecto en el tiempo ${ }^{85}$.

- Curcumina: es un producto de origen vegetal con efecto antioxidante que, asociado a metformina, tendría un efecto cardioprotector. Los estudios se encuentran en fase experimental y necesitan más investigación ${ }^{86,87}$.

- Análogos de GLP-1: no se dispone de estudios que consideren sus efectos en DM1 y ECV88.

- Inhibidores SGLT-2: hasta el momento no hay estudios en DM1 y ECV, sin embargo, aquellos que se realizaron en este grupo de pacientes con estos fármacos mostraron resultados en variabilidad glucémica y menor requerimiento de insulina ${ }^{65}$.

- Antiagregación: el ácido acetil salicílico demostró beneficios en prevención secundaria; en prevención primaria, considerar en casos especiales con DM1 y múltiples FRCV, y evaluar el balance entre riesgo $\mathrm{CV}$ y riesgo hemorrágico ${ }^{65}$.

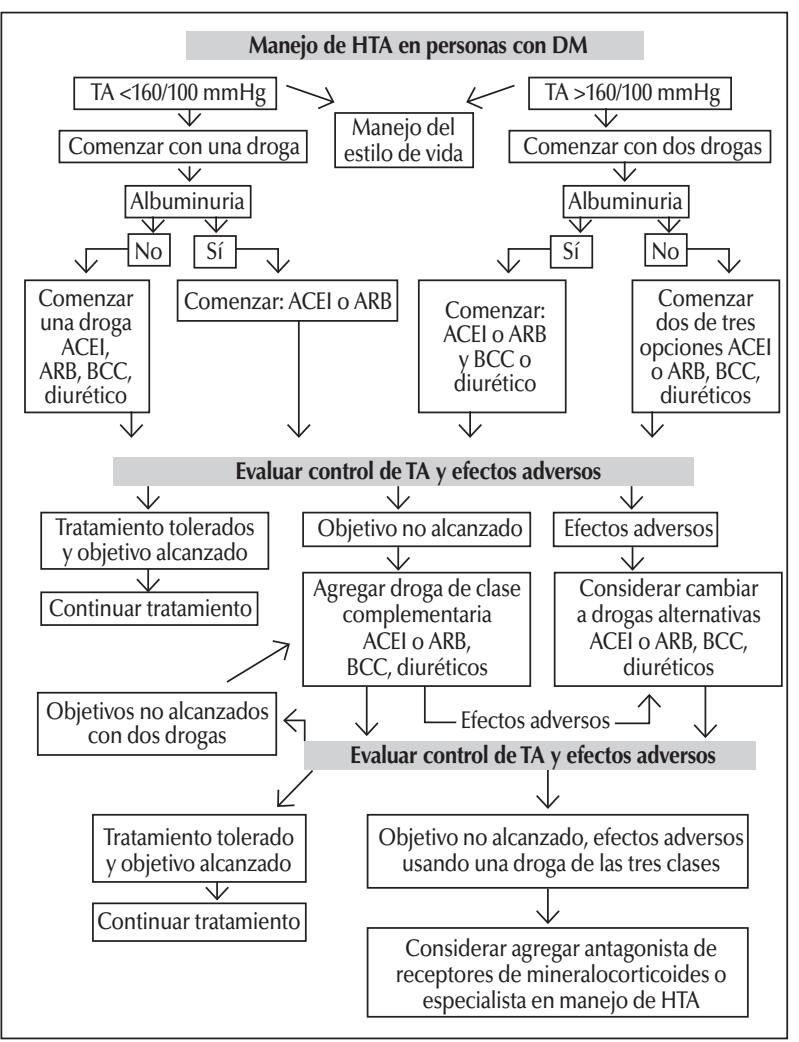

Fuente: Consenso Argentino de Hipertensión Arteria/ ${ }^{80}$.

Figura 3: Recomendaciones para el manejo de la HTA en personas con $\mathrm{DM}^{80}$. 


\section{DM1 y ECV en poblaciones especiales \\ - Embarazo}

En toda mujer con DM1 se recomienda programar su embarazo, y se desaconseja ante enfermedad coronaria no revascularizada, insuficiencia renal, retinopatía proliferativa activa sin respuesta a la panfotocoagulación y en HTA que no mejora con fármacos permitidos en el embarazo. Una mujer con DM1 y enfermedad cardiovascular previa tendrá un riesgo muy elevado de complicaciones fetales, con riesgo de sobrevida fetal. Asimismo, la DM1 per se es un factor de riesgo para desarrollar complicaciones hipertensivas, en particular preeclampsia, con una frecuencia 3-7 veces mayor, aumentando su incidencia en presencia de nefropatía y/o retinopatía diabética proliferativa. La preeclampsia se asocia con mayor riesgo de hipertensión arterial a futuro, con aumento del riesgo cardiovascular y es un factor de riesgo independiente para la morbilidad cardiovascular a largo plazo de su descendencia7,89,90,91,92,93.

\section{- Niños y adolescentes}

La ateroesclerosis comienza a edades tempranas de la vida. Se estima que entre el $14-45 \%$ de los niños con DM1 tienen dos o más factores de riesgo cardiovascular como mayor $\mathrm{HbA} 1 \mathrm{c}$, obesidad, TA $\geq$ percentilo 90 , colesterol total $>200 \mathrm{mg} / \mathrm{dl}$ y LDL $>130 \mathrm{mg} / \mathrm{dl}$. Éstos aumentan con la edad y el sexo, y con mayor riesgo en niñas que en niños, las cuales suelen tener niveles de LDL más altos y HDL más bajo. A pesar de la presencia frecuente de HTA y dislipemia, una baja proporción de estos niños recibe tratamiento farmacológico. Asimismo, durante la primera década posterior al diagnóstico de DM1, puede encontrarse ECV subclínica. En base a ello, se ha propuesto detectar placas carotídeas en niños y adolescentes con DM1, y se encontró correlación con la duración de la DM, el IMC, colesterol total, LDL, tabaquismo, HTA y excreción urinaria de albumina; sin embargo otros autores no mostraron diferencias con respecto a la población general, por lo cual la evidencia sobre dicho método resulta aún poco concluyente $7,79,94,95,96,97,97,99,100,101$.

Al evaluar marcadores de enfermedad cardiovascular en la población pediátrica, se recomienda realizar screening de TA y dislipemia, según normas de la International Society for Pediatric and Adolescent Diabetes (ISPAD) 2018 ${ }^{102}$. De acuerdo a dicho consenso, así como lo que plantea la American Diabetes Association (ADA) y la guía de práctica clínica para el manejo de DM1 (Sociedad Argentina de Diabetes), se propone medir laTA en cada consulta, y establecer HTA ante TA sistólica o diastólica $\geq$ percentilo 95 para edad, sexo y altura, en tres o más ocasiones. El tratamiento consistirá en modificaciones en el estilo de vida y, si pasados los 3-6 meses no se logran los objetivos, se iniciarán fármacos como IECA o ARA II. Se recomienda no omitir el consejo reproductivo.

En cuanto a la dislipemia, en la Tabla 6 se describe cuándo solicitar un perfil lipídico, objetivos lipídicos y tratamiento en niños y adolescentes, según normas de la ISPAD 2018, ADA y la Sociedad Argentina de Pediatría. Si bien no hay estudios a largo plazo sobre la eficacia de las estatinas en la población pediátrica, en base a estudios a corto plazo, principalmente en contexto de hipercolesterolemia, datos en adultos y recientes resultados del estudio Adolescent type 1 Diabetes cardiorenal Intervention Trial (AdDit) sobre la seguridad de atorvastatina en adolescentes, se establecen recomendaciones en el uso de estatinas ${ }^{79,102,103,104,105}$.

La presencia de albuminuria también es otro factor de riesgo cardiovascular. En adolescentes con DM tipo 1 de 10-16 años de edad se ha visto que, aún con excreciones urinarias de albumina/ creatinina en el límite superior de lo normal, presentan peor evolución cardiovascular con más HTA, engrosamiento intimal carotideo y parámetros inflamatorios más elevados (PCR) luego de 2-4 años de seguimiento ${ }^{106}$. 
Revista de la Sociedad Argentina de Diabetes Vol. 54 № 2 Suplemento I Jornadas Conjuntas del Comité de Graduados y el Comité de Hipertensión Arterial y otros FRCV. XVIII Jornadas del Comité de Graduados

Mayo-agosto de 2020: 71-90 ISSN 0325-5247 (impresa) ISSN 2346-9420 (en línea)

\begin{tabular}{|c|c|c|}
\hline & ¿Cuándo realizar screening? & Objetivos y tratamiento \\
\hline ADA 2019 & $\begin{array}{l}\text { - Perfil lipídico en niños >10 años al diagnóstico de DM una } \\
\text { vez controlada } \\
\text { - A los } 2 \text { años de edad si antecedentes familiares de ECV } \\
\text { prematura o historia de hipercolesterolemia familiar }\end{array}$ & $\begin{array}{l}\text { - } \mathrm{LDL}<100 \mathrm{mg} / \mathrm{dl} \text { : repetir cada 3-5 años } \\
\text { - } \mathrm{LDL}>100 \mathrm{mg} / \mathrm{dl} \text { : optimizar control glucémico o plan ali- } \\
\text { mentario (grasas saturadas }<7 \% \text { del VCT, colesterol } \\
<200 \mathrm{mg} / \mathrm{dl} \text { ) } \\
\text {-Estatinas: niño }>10 \text { años y persistencia de } \mathrm{LDL}>160 \mathrm{mg} / \mathrm{dl} \\
\text { a pesar de tratamiento nutricional } \\
\text { o LDL }>130 \mathrm{mg} / \text { dl y otro FRCV }\end{array}$ \\
\hline ISPAD 2018 & $\begin{array}{l}\text { - Perfil lipídico en niños >11 años al diagnóstico de DM una } \\
\text { vez estabilizada } \\
\text { - Si valores normales, repetir cada cinco años } \\
\text { - A los } 2 \text { años de edad si antecedentes familiares de ECV } \\
\text { prematura o historia de hipercolesterolemia }\end{array}$ & $\begin{array}{l}\text { - } \mathrm{LDL}>100 \mathrm{mg} / \mathrm{dl} \text { : cambios de estilo de vida } \\
\text { - Niños }>10 \text { años y } \mathrm{LDL}>130 \mathrm{mg} / \mathrm{dl} \text { a pesar de cambios en } \\
\text { estilo de vida: estatinas }\end{array}$ \\
\hline $\begin{array}{l}\text { Sociedad } \\
\text { Argentina } \\
\text { de Pediatría } \\
2015\end{array}$ & $\begin{array}{l}\text { Independientemente de la edad, si el niño tiene anteceden- } \\
\text { tes familiares o riesgo de ECV como DM }\end{array}$ & $\begin{array}{l}\text { - } \mathrm{LDL}<110 \mathrm{mg} / \mathrm{dl} \text { : aceptable } \\
\text { - Esencial el plan nutricional } \\
\text { - Tratamiento farmacológico: } \\
\text {. Niños }>8-10 \text { años y } \mathrm{LDL}>190 \mathrm{mg} / \mathrm{dl} \\
\text {. Niños }>8-10 \text { años con } \mathrm{LDL}>160 \mathrm{mg} / \mathrm{dl} \text { y hasta dos FRCV } \\
\text { agregados } \\
\text {. Niños }>10 \text { años con C-LDL }>130 \mathrm{mg} / \mathrm{dl} \text { y más de dos FRCV } \\
\text { agregados }\end{array}$ \\
\hline
\end{tabular}

Fuente: Propuesta del Comité de Graduados para las Jornadas.

ECV: enfermedad cardiovascular; VCT: valor calórico total; FRCV: factores de riesgo cardiovascular.

Tabla 6: Evaluación de la dislipemia en población pediátrica con DM.

Por otro lado, el tabaquismo se asocia con mayor riesgo de presentar albuminuria en forma persistente e incrementa la morbimortalidad cardiovascular de estos pacientes por lo cual será esencial el cese tabáquico en esta población. Asimismo, es preocupante el inicio temprano del consumo de bebidas alcohólicas en adolescentes, generalmente en forma excesiva y episódica. Según datos de un estudio en colegios secundarios, la edad promedio del inicio de consumo de alcohol es a los 13 años, lo cual impactará en el desarrollo posterior de dependencia, trastornos metabólicos asociados como hipertigliceridemia, enfermedad hepática, mayor riesgo cardiovascular y hasta muerte ${ }^{102,107}$.

La enfermedad ateroesclerótica se inicia en la vida infanto juvenil y aquellos con DM1 tienen mayor riesgo cardiovascular y hasta una mortalidad por dicha causa hasta 13 veces mayor que la población general, ya detectable desde la segunda década de la vida. Por ello, será esencial la detección y tratamiento precoz de dichos factores ya que la infancia y la adolescencia son períodos durante los cuales podrán prevenirse o retrasar el inicio y progresión de las complicaciones ${ }^{108}$.

\section{- Adultos mayores}

La esperanza de vida de las personas con DM1 ha mejorado considerablemente en las últimas décadas debido al mejor manejo de factores glucémicos y no glucémicos. Sin embargo, pese a la reducción del riesgo relativo de ECV, la morbilidad y mortalidad aún son inaceptablemente altas para esta población. La edad de inicio de la DM1 es un determinante importante de la supervivencia, y el enfoque en la cardioprotección debe ser mayor en personas con DM1 de inicio temprano y edad avanzada $8,109,110$.

La prevalencia de ECV en personas adultas mayores con DM1 es de aproximadamente $40 \%$. En el Scottish Registry Linkage Study (SRLS) la tasa de incidencia relativa del primer evento de ECV para pacientes mayores de 70 años fue de 1,71 para hombres y 1,85 para mujeres, y en el United Kingdom General Practice Research Database (GPRD) el HR para el primer evento CV para el grupo de edad de 65 a 75 años fue de 2,3 para los hombres y 8,3 para las mujeres ${ }^{25,111,112}$.

Como se expuso previamente, las guías actuales proponen que el screening y el manejo de DM1 y ECV debería ser similar al de DM2, pero en los adultos mayores habrá que incluir requisitos adicionales y estratificarlos para fijar objetivos de tratamiento según estado de dependencia, cognición, funcionalidad y capacidad de automanejo de la diabetes ${ }^{11,65,113,114,115}$.

Entre los FRCV tradicionales modificables que deben detectarse y tratarse se mencionan: obesidad, sedentarismo, tabaquismo, alcoholismo, periodontitis, depresión, control glucémico subestimo, dislipemia, HTA, enfermedad renal crónica, albuminuria, depresión, apnea del sueño y fibrila- 
ción auricular, y entre los no modificables: edad, tiempo desde el diagnóstico, etnia, sexo, situación socioeconómica y antecedentes familiares de ECV ${ }^{114}$ (Tabla 7).

En subpoblaciones especiales con DM1 de larga evolución (Medallistas Joslin, EE.UU.) y Cohorte de los Años Dorados (United Kingdom awards) se describen factores protectores de ECV: insulinosensibilidad (promedio 0,52 U/kg/ día de insulina), HbA1c de 7,6\%, no nefropatía pese a presencia de albuminuria y función residual de péptido C. Estas características son consistentes con un estado de sensibilidad a la insulina conservado ${ }^{116,117 .}$

\section{Trasplante reno pancreático}

El trasplante reno pancreático (TRP) es el tratamiento de elección en pacientes con DM1 e insuficiencia renal crónica. La supervivencia en lista de espera de los pacientes con DM que permanecen en diálisis es de aproximadamente $58 \%$ a cuatro años vs $90 \%$ de aquellos que se beneficiaron delTRP en el mismo período. La supervivencia a largo plazo del paciente y del injerto renal mejora con el trasplante. EI TRP parece también mejorar las complicaciones microvasculares, la función cardiovascular y el grosor de la íntima media carotídea, el perfil lipídico y la tensión arterial. Ensayos no aleatorios sugieren una reducción en la mortalidad CV118,119,120.

\begin{tabular}{|c|c|c|c|}
\hline & $\begin{array}{l}\text { Categoría } 1 \\
\text { Totalmente independiente }\end{array}$ & $\begin{array}{l}\text { Categoría } 2 \\
\text { Parcialmente independiente }\end{array}$ & $\begin{array}{l}\text { Categoría } 3 \\
\text { Dependiente }\end{array}$ \\
\hline Manejo de FRCV & $\begin{array}{l}\text { Aplica manejo y tratamiento de } \\
\text { todos los ítems de los FRCV }\end{array}$ & $\begin{array}{l}\text { Considerar objetivos apropiados } \\
\text { para aquellos cuya salud } \\
\text { funcional está irreversiblemente } \\
\text { comprometida }\end{array}$ & $\begin{array}{l}\text { Al final de la vida, el control de } \\
\text { FRCV no es necesario e incluso el } \\
\text { retiro de terapias innecesarias puede } \\
\text { ser apropiado }\end{array}$ \\
\hline $\begin{array}{l}\text { Control glucémico } \\
\text { preprandial } \\
>90-126 \mathrm{mg} / \mathrm{dl} \\
\text { Postprandial }<180 \mathrm{mg} / \mathrm{dl} \text { ) }\end{array}$ & $\begin{array}{l}\text { HbA1c } 6,5-7 \% \\
>\text { de } 70 \text { años HbA1c } 7 \text { a } 7,5 \%\end{array}$ & HbA1c $7,5-8 \%$ & $\begin{array}{l}\text { Objetivo individualizado } \\
\text { Evitar hiperglucemia sostenida } \\
\mathrm{HbA} 1 \mathrm{c}<8,5 \%\end{array}$ \\
\hline Hipoglucemias & \multicolumn{3}{|l|}{ Evitar } \\
\hline Automonitoreo & $\begin{array}{l}\text { Mínimo preprandial } \\
\text { MCG si estuviera indicado }\end{array}$ & \multicolumn{2}{|l|}{ Evitar regímenes complejos } \\
\hline $\begin{array}{l}\text { Objetivo de TA } \\
<140 \mathrm{mmHg} \text { si es bien } \\
\text { tolerada. Nunca } \\
<120 \mathrm{mmHg} \\
\text { Buscar hipotensión } \\
\text { ortostática }\end{array}$ & $\begin{array}{l}<140 / 80 \\
\text { Primera opción: IECA/ARAll } \\
\text { Betabloqueantes, antagonistas } \\
\text { canales de calcio y diuréticos según } \\
\text { comorbilidades }\end{array}$ & $<140 / 80$ & $\begin{array}{l}\text { 150/90 (en demencia no avanzada } \\
\text { un objetivo menor puede mejorar } \\
\text { estado cognitivo restante) }\end{array}$ \\
\hline $\begin{array}{l}\text { Objetivos dislipemia } \\
\mathrm{LDL}<70 \text { o }<100 \mathrm{mg} / \mathrm{dl}, \\
\text { según estratificación de } \\
\text { riesgo } \\
\text { Triglicéridos }<200 \mathrm{mg} / \mathrm{dl}\end{array}$ & $\begin{array}{l}\text { Gestión activa para alcanzar los } \\
\text { objetivos } \\
\text { Estatinas terapia de primera línea } \\
\text { Fibratos en caso de TG >800 mg/dl }\end{array}$ & $\begin{array}{l}\text { Intervenciones según } \\
\text { comorbilidades con objetivos } \\
\text { individualizados }\end{array}$ & $\begin{array}{l}\text { El umbral para diagnóstico y trata- } \\
\text { miento debe ser alto } \\
\text { Retirar tratamientos puede ser apro- } \\
\text { piado (evidencias contradictorias en } \\
\text { demencia) }\end{array}$ \\
\hline $\begin{array}{l}\text { No HDL }<97 \mathrm{mg} / \mathrm{dl} \\
\mathrm{LDL}<70 \mathrm{mg} / \mathrm{dl} \text { en ECV } \\
\text { establecida }\end{array}$ & \multicolumn{3}{|c|}{ Atención sobre efectos adversos del tratamiento farmacológico } \\
\hline
\end{tabular}

Fuente: Sinclair AJ, et al. ${ }^{114}$.

FRCV: factores de riesgo cardiovascular; MCG: monitoreo continuo de glucosa; TA: tensión arterial; TG: triglicéridos; ECV: enfermedad cardiovascular.

Tabla 7: Categorías de pacientes ancianos según funcionalidad y ECV.

\section{Rehabilitación cardiovascular en DM1}

La rehabilitación cardíaca $(\mathrm{RC})$ ha sido reconocida como un componente integral del tratamiento para los pacientes que sufrieron un evento cardiovascular. Los objetivos de estos programas son reducir la discapacidad secundaria a la enfermedad cardiovascular, disminuir el riesgo de nuevos eventos y la mortalidad. El ejercicio regular y la adaptación al estilo de vida cardiovascular se han convertido en un problema importante en el tratamiento cardiovascular en un entorno de prevención primaria y secundaria ${ }^{121,122,123 .}$

En prevención secundaria, variables clínicas como la mortalidad cardiovascular y la mortalidad global, así como los eventos cardiovasculares recurrentes y las hospitalizaciones fueron significativamente más bajas en pacientes evaluados posterior a la $\mathrm{RC}^{124,125}$. 


\section{Beneficios de la RC en DM1}

En prevención primaria, un subestudio del Prospective Urban Rural Epidemiology (PURE) demostró recientemente que el ejercicio regular es capaz de reducir los eventos CV en individuos que siguen un entrenamiento regular independientemente de otros factores de riesgo cardiovascular. Además, estos beneficios siguen una "relación dosis-efecto", en particular en lo que respecta al ejercicio ("cuanto más mejor") ${ }^{126}$.

Otro hallazgo significativo de la presente revisión sistemática es la baja tasa de participación en RC en personas con DM. Una limitación de los estudios identificados es que en los mismos no se refieren si los participantes tenían DM1 o DM2. Por lo tanto, se necesita más investigación para demostrar cualquier diferencia entre distintos tipos de $\mathrm{DM}^{127}$.

Los diagnósticos elegibles para rehabilitación cardiovascular incluyen:

- Infarto agudo de miocardio en los 12 meses anteriores.

- Cirugía de bypass de arteria coronaria.

- Angina de pecho estable actual.

- Reparación o reemplazo de la válvula cardíaca (cirugía abierta o de cateterismo).

- Angioplastia coronaria transluminal percutánea o stent coronario.

- Trasplante de corazón o corazón/pulmón.

- Insuficiencia cardíaca crónica estable con fracción de eyección reducida (FEy $\leq 35 \%$ ).

\section{CONCLUSIONES}

- El riesgo de muerte por cualquier causa y de causa cardiovascular en DM1 con control glucémico subóptimo es ocho a 10 veces mayor que en la población general.

- La edad de inicio y el tiempo desde el diagnóstico de la DM1 son determinantes cruciales para la ECV.

- Las mujeres con DM1 tienen 40\% más de riesgo de mortalidad por todas las causas y el doble de riesgo de eventos $\mathrm{CV}$ fatales y no fatales en comparación a sus pares masculinos.

- La fisiopatología de la ECV en personas con DM1 es compleja y está parcialmente dilucidada.

- En la DM1 existiría un entorno procoagulante/hipofibrinolítico que conduciría a una mayor morbimortalidad.

- Los factores de riesgo tradicionales (edad, edad de inicio de DM1, sexo femenino, duración de diabetes, HbA1c, variabilidad glucémica, albu-
minuria-VFGe y PAS) parecen funcionar de manera diferente en DM1.

- No existen calculadoras de riesgo cardiovascular validadas en DM1.

- En la clasificación de riesgo cardiovascular en DM1 no existe el riesgo bajo.

- La detección de ECV subclínica en pacientes con DM1 es controvertida y no se recomienda en forma sistemática.

- La afección miocárdica comienza tempranamente y es una complicación frecuente en personas con DM1, pudiéndose desarrollar CMD diastólica subclínica antes de la disfunción sistólica y aún antes de los síntomas clínicos.

- La disfunción diastólica se presenta como el primer signo de CMD, hallándose como un marcador, y debería tomárselo en cuenta como FRCV para identificar pacientes con alto riesgo de IC y muerte cardiovascular.

- El ACV es una complicación frecuente en las personas con DM1 y se presenta en etapas más tempranas de la vida que en aquellas sin diabetes.

- El tratamiento no farmacológico se asemeja al de las personas con DM2 en el marco de programas educativos formales con abordaje multidisciplinario. La actividad física es fuertemente recomendada. El plan alimentario no difiere del indicado en DM2, excepto al considerar un mayor manejo en el conteo de hidratos de carbonos y el índice glucémico.

- Promover el cese tabáquico y desalentar el exceso de consumo de alcohol.

- En la población pediátrica y adolescentes con DM1 es esencial la detección y tratamiento precoz de FRCV a fin de prevenir o retrasar el inicio y progresión de eventos CV.

- La evaluación del riesgo CV en el adulto mayor debe ser individualizado y categorizarlo según funcionalidad y comorbilidades a fin de fijar objetivos de control de factores glucémicos y no glucémicos personalizados.

\section{BIBLIOGRAFÍA}

1. Orchard TJ, Costacou T, Kretowski A. et al. Type 1 diabetes and coronary artery disease. Diabetes Care 2006; 29:2528-2538.

2. Lind M, Svensson AM, Kosiborod M, et al. Glycemic control and excess mortality in type 1 diabetes. NEJM 2014; 371:1972-82.

3. Pober D, Galderisi A, HuiJuan L, et al. Glycemic control, cardiac autoimmunity and long-term risk of cardiovascular disease in type 1 diabetes mellitus: A DCCT/EDIC cohort-based study. Circulation 2018; 138:00-14. 
4. Miller RG, Costacou T, Orchard TJ, et al. Risk factor modeling for cardiovascular disease in type 1 diabetes in the Pittsburgh Epidemiology of Diabetes Complications (EDC) Study. A comparison to the Diabetes Control and Complications Trial/ Epidemiology of Diabetes Interventions and Complications Study. Diabetes 2018;1-27.

5. Soedamah-Muthu SS, Fuller JH, Mulnier HE, et al. High risk of cardiovascular disease in patients with type 1 diabetes in the U.K.: a cohort study using the general practice research database. Diabetes Care 2006; 29:798-804.

6. Secrest AM, Becker DJ, Kelsey SF, Laporte RE, Orchard TJ. Cause-specific mortality trends in a large population-based cohort with longstanding childhood-onset type 1 diabetes. Diabetes 2010; 59:3216-3222.

7. De Ferranti SD, de Boer IH, Fonseca V, et al. Type 1 Diabetes mellitus and cardiovascular disease. A scientific statement from the American Heart Association and American Diabetes Association. Diabetes Care 2014; 37(10):2843-2863. DOI:10.2337/dc14-1720.

8. Rawshani A, Sattar N, Franzén S, et al. Excess mortality and cardiovascular disease in young adults with type 1 diabetes in relation to age at onset: a nationwide, register-based cohort study. Lancet 2018; 392:477-86. DOI:10.1016/S0140-6736(18)31506-X.

9. Rawshani A, Sattar N, Franzen S, et al. Relative prognostic importance and optimal levels of risk factors for mortality and cardiovascular outcomes in type 1 diabetes mellitus. Circulation 2019;139(6):1900-1012. DOI:10.1161/CIRCULATIONAHA. 118.037454 .

10. Schofield J, Ho J, Soran H. Cardiovascular risk in type 1 diabetes mellitus. Diabetes Ther 2019; 10:773-789. DOI:10.1007/ s13300-019-0612.

11. Cosentino F, Grant PJ, Aboyans V, et al. ESC Guidelines on diabetes, pre-diabetes and cardiovascular diseases developed in collaboration with the EASD. Eur Heart J 2020; 41(2):255-323. DOI:10.1093/eurheartj/ehz486.

12. Htay T, Soe K, López-Pérez A, et al. Mortality and cardiovascular disease in type 1 and type 2 diabetes. Curr Cardiol Rep. 2019;21(6):45. Published 2019 Apr 22. DOI:10.1007/s11886-019$1133-9$.

13. Huxley RR, Peters SA, Mishra GD, et al. Risk of all-cause mortality and vascular events in women versus men with type 1 diabetes: a systematic review and meta-analysis. Lancet Diabetes Endocrinol 2015; 3:198-206. DOI:10.1016/S2213-8587(14)70248-7.

14. Peters SA, Huxley RR, Woodward M, et al. Diabetes as risk factor for incident coronary heart disease in women compared with men: a systematic review and meta-analysis of 64 cohorts including 858,507 individuals and 28,203 coronary events. Diabetologia 2014; 57:1542-51. DOI:10.1007/s00125-014-3260-6.

15. Roy M, Rendas-Baum R, Skurnick J. Mortality in African-Americans with type 1 diabetes: the New Jersey 725. Diabet Med 2006; 23(6):698-706. DOI:10.1111/j.1464-5491.2006.01901.x.

16. Bosnyak Z, Nishimura R, Hagan-Hughes M, et al. Excess mortality in black compared with white patients with type 1 diabetes: an examination of underlying causes. Diabet Med 2005; 22:1636-1641

17. Walsh MG, Zgibor J, Borch-Johnsen K, Orchard TJ; DiaComp Investigators. A multinational assessment of complications in type 1 diabetes: the DiaMond substudy of complications (DiaComp) level 1. Diab Vasc Dis Res 2006; 3:84-92.

18. Nathan DA, Cleary PA, Jye-Yu MS, Backlund C, et al. Intensive diabetes treatment and cardiovascular disease in patients with type 1 diabetes. NEJM 2005 353; 2643-2653. DOI:10.1056/NEJMoa052187.

19. Soedamah-Muthu SS, Chaturvedi N, Witte DR, Stevens LK, Porta $\mathrm{M}$, et al. Relationship between risk factors and mortality in type 1 diabetic patients in Europe. The EURODIAB Prospective Complications Study (PCS). Diabetes Care 2008; 31(7):13601366. DOI:10.2337/dc08-0107.

20. Klein BE, Klein R, McBride PE, Cruickshanks KJ, et al. Cardiovascular disease, mortality, and retinal microvascular characteristics in type 1 diabetes. Arch Intern Med 2004; 164(17):19171924. DOI:10.1001/archinte.164.17.1917.
21. Dabelea D, Kinney G, Snell-Bergeon JK, Hokanson JE, et al. Effect of type 1 diabetes on the gender difference in coronary artery calcification: a role for insulin resistance? The Coronary Artery Calcification in Type 1 Diabetes (CACTI) Study. Diabetes. 2003; 52(11):2833-2839. DOI:10.2337/diabetes.52.11.2833.

22. Pease AJ, Earnest A, Nanayakkara N, Ranashina S et al. Burden of cardiovascular risk factors and disease in type 1 diabetes. Results of the Australian National Diabetes Audit (ANDA). Cardiovasc Diabetol. 2018;17(1):77. Published 2018 Jun 2. DOI:10.1186/ s12933-018-0726-8 .

23. Lind M, Pivodi A, Svensson AM, Ólafsdóttir AF, et al. HbA1c level as a risk factor for retinopathy and nephropathy in children and adults with type 1 diabetes: Swedish population based cohort study. British Medical Journal; 2019.366:14894. Published 2019 Aug 28. DOI:10.1136/bmj.14894.

24. Kiss Z, Rokszin G, Abonyi-Tóth Z, Jermendy G, et al. Young adult patients with type 1 diabetes have a higher risk of mortality than those of similar age with type 2 diabetes: A nationwide analysis in Hungary. Diabetes Metab Res Rev 2019; 35:e3190.

25. Livingstone SJ, Looker HC, Hothersall EJ, Wild SH. Risk of cardiovascular disease and total mortality in adults with type 1 diabetes: Scottish Registry Linkage Study. PLoS Medicine 2012; 9(10): e1001321. DOI:10.1371/journal.pmed.1001321.

26. Steineck I, Cederholm J, Eliasson B, Rawshani A, et al. Insulin pump therapy, multiple daily injections, and cardiovascular mortality in 18,168 people with type 1 diabetes: observational study. British Medical Journal 2015; 350:h3234. Published 2015 Jun 22. DOI:10.1136/bmj.h3234.

27. Brown RA, Shantsila E, Varma C, et al. Current understanding of atherogenesis. Am J Med. 2017; 130(3):268-282. DOI:10.1016/j. amjmed.2016.10.022.

28. Costacou T, Lopes-Virella MF, Zgibor JC, et al. Markers of endothelial dysfunction in the prediction of coronary artery disease in type 1 diabetes: the Pittsburgh Epidemiology of Diabetes Complications Study. J Dia Complic 2005; 19:183-193.

29. Faxon $D$, Fuster $V$, Libby $P$, et al. Atherosclerotic vascular disease conference. Circ 2004; 109 (21):2617-2625.

30. Cooper ME, El-Osta A, Allen TJ, et al; Metabolic Karma. The atherogenic legacy of diabetes:The 2017 Edwin Bierman Award Lecture. Diab 2018; 67:785-790.

31. Pechlivani N, Ajjan RA. Thrombosis and vascular inflammation in diabetes: mechanisms and potential therapeutic targets. Front Cardiovasc Med 2018; 19,5:1. DOI: 10.3389/ fcvm.2018.00001.

32. Costacou T, Ferrell RE, Orchard TJ et al. Haptoglobin genotype: a determinant of cardiovascular complication risk in type 1 diabetes. Diabetes 2008; 57:1702-1706.

33. Reddy MA, Zhang E, Natarajan R. Epigenetic mechanisms in diabetic complications and metabolic memory. Diabetologia 2015; 58:443-455.

34. Khunti K, Davies M, Majeed A, et al. Hypoglycemia and risk of cardiovascular disease and all-cause mortality in insulintreated people with type 1 and type 2 diabetes: a cohort study. Diabetes Care 2015; 38(2):316-22.

35. Mellbin LG, Ryden L, et al. Does hypoglycaemia increase the risk of cardiovascular events? A report from the ORIGIN trial. Eur Heart J 2013; 34(40):3137-44.

36. Hoffman RP, Dye AS, Huang $H$, et al. Glycemic variability predicts inflammation in adolescents with type 1 diabetes. J Pediatr Endocrinol Metab 2016; 29(10):1129-1133.

37. Larkin ME, Backlund JY, Cleary $\mathrm{P}$, et al. Disparity in management of diabetes and coronary heart disease risk factors by sex in DCCT/EDIC. Diabet Med 2010; 27(4):451-8.

38. Petrie $D$, Lung TWC, Rawshani $A$, et al. Recent trends in life ex pectancy for people with type 1 diabetes in Sweden. Diabetologia 2016; 59:1167-1176. DOI 10.1007/s00125-016-3914-7.

39. Thorn LM, Forsblom C, Fagerudd J, Thomas M, et al. Metabolic syndrome in type 1 diabetes. Association with diabetic nephropathy and glycemic control (the FinnDiane study). Diabetes Care 2005; 28 (8):2019-24. 
Revista de la Sociedad Argentina de Diabetes Vol. 54 № 2 Suplemento I Jornadas Conjuntas del Comité de Graduados y el Comité de Hipertensión Arterial y otros FRCV. XVIII Jornadas del Comité de Graduados

Mayo-agosto de 2020: 71-90 ISSN 0325-5247 (impresa) ISSN 2346-9420 (en línea)

40. Ing Lee S, Patel M, Jones CM, Narendran P. Cardiovascular disease and type 1 diabetes: prevalence, prediction and management in an ageing population. Ther Adv Chronic Disv 2015 Nov; 6(6): 347-374.

41. Kramer CK, RodriguesTC, Canani LH, et al. Diabetic retinopathy predicts all-cause mortality and cardiovascular events in both type 1 and 2 diabetes: meta-analysis of observational studies. Diabetes Care 2011; 34(5):1238-44. https://doi.org/10.2337/dc110079.

42. Zobel EH, Hasbak P, Winther SA, et al. Cardiac autonomic function is associated with myocardial flow reserve in type 1 diabetes. Diabete 2019; 68(6):1277-1286.

43. Terrasa S, Buela G, Guenzelovich T, Sigal T, et al. Biomarkers and clinical findings in the prediction of cardiovascular risk: novel risk factors? Disponible en: https://www.researchgate.net/publication/282133785_Marcadores_serologicos_y_clinicos_en_la_prediccion_del_riesgo_cardiovascular_nuevos_factores_de_riesgo-.

44. Ortega E, Amor AJ, Rojo-Martínez G, Castell C, et al. Enfermedad cardiovascular en pacientes con diabetes mellitus tipo 1 y tipo 2 en España. Medicina Clínica (Barcelona) 2014; 3160:1-6.

45. Michael E. Vascular disease in the lower limb in type 1 diabetes. Cardiovascular Endocrinology and Metabolism 2019; 8:39-46.

46. De la Hera JM, García-Ruiz JM, Delgado E. Diabetes y cribado de enfermedad coronaria: ¿dónde centramos el esfuerzo? Revista Española Cardiología 2015; 68:830-833.

47. Layerle $B$, Vignolo W. Tamizaje de cardiopatía isquémica y revascularización en pacientes diabéticos con enfermedad arterial coronaria estable. Articulo de revisión. Revista Uruguaya de Cardiología 2017; Vol 32, 2:158-172. Disponible en: http:// www.scielo.edu.uy/scielo.php?script=sci_arttext\&pid=S168804202017000200158\&Ing=es.

48. Levre MM, Moulin P, Thivolet C, Rodier M, Rigalleau V, Penfornis $A$, et all. Detection of silent myocardial ischemia in asymptomatic patients with diabetes: results of randomized Trial and metanalysis assessing in efectiveness of systematic screening. Trials 2011; 26:12-23.

49. Marso SP, Hiatt WR. Peripheral arterial disease in patients with diabetes. Journal of the American College of Cardiology 2006; 47(5):921-929.

50. Tehan PE, Chuter VH, et al. How sensitive and specific is continous- wave Doppler for detecting peripheral arterial disease in people with and without diabetes? A cross-sectional study. Diabetes \& Vascular Disease Research 2018;15:396-401.

51. Buitrago-Ramirez F, Pérez-Caballero FL. Arteriopatia periférica. Formación Médica Continuada en Atención Primaria 2013; 20(5):246-255

52. Sundquist K, Li X. Type 1 diabetes as a risk factor for stroke in men and women aged 15-49: a nationwide study from Sweden. Diabet Med 2006; 23:1261-1267. DOI:10.1111/j.14645491.2006.01959.x.

53. Putaala $R$, Liebkind $D$, et al. Diabetes mellitus and ischemic stroke in the young. Neurología 2011; 76:1831-1837. DOI:10.1212/ WNL.0b013e31821cccc2.

54. Chen R, Ovbiagele B, et al. Diabetes and stroke: epidemiology, pathophysiology, pharmaceuticals and outcomes. Am J Med Sci 2016 April; 351(4):380-386.

55. Janghorbani M, Hu FB, et al. Prospective study of type 1 and type 2 diabetes and risk of stroke subtypes. Diabetes Care 2007; 30:1730-1735. DOI:10.2337/dc06-236.

56. Heden-Stahl C, Lind M, Svensson AM, et al. Glycaemic control and excess risk of ischaemic and haemorrhagic stroke in patients with type 1 diabetes: a cohort study of 33453 patients. J Intern Med 2017 Mar; 281(3):261-272. DOI: 10.1111/joim.12572.

57. Hägg S, Thorn LM. Different risk factor profiles for ischemic and hemorrhagic. stroke in type 1 diabetes mellitus. Stroke 2014; 45:2558-2562.

58. Hagg-Holmberg S, Thorn LM, Forsblom CM, et al. Prognosis and its predictors after incident stroke in patients with type 1 diabetes. Diabetes Care 2017; 40:1394-1400.
59. Hagg-Holmberg S, Dahlström EH, Forsblom CM, et al. The role of blood pressure in risk of ischemic and hemorrhagic stroke in type 1 diabetes. Cardiovasc Diabetol 2019; 18:88.

60. Hagg S, Lena M. Thorn, Putaala J, et al. Incidence of stroke according to presence of diabetic nephropathy and severe diabetic retinopathy in patients with type 1 diabetes. Diabetes Care 2013; 36:4140-4146.

61. Seferovic PM, Paulus WJ. Clinical diabetic cardiomyopathy: a two-faced disease with restrictive and dilated phenotypes. European Heart Journal 2015; 36:1718-1727. DOI:10.1093/eurheartj/ehv134.

62. Hölscher ME, Bode C, Bugger H. Diabetic cardiomyopathy: Does the type of diabetes matter? Int J Mol Sci 2016; 17: 2136.

63. Lind M, Bounias I,Olsson M, Gudbjörnsdottir S, et al. Glycaemic control and incidence of heart failure in 2.985 patients with type 1 diabetes: an observational study. Lancet 2011; 378:140-46.

64. Gulsin GS, Athithan L, McCann GP. Diabetic cardiomyopathy: prevalence, determinants and potential treatments. Ther Adv Endocrinol Metab 2019; Vol. 10: 1-21.

65. American Diabetes Association. Standards of Medical Care in Diabetes. Chapter 10 Cardiovascular disease and risk management. Diabetes Care 2019; 42(S1): S103-123. DOI:10.2337/ dc19-S010.

66. American Diabetes Association. Standards of Medical Care in Diabetes. Chapter 5: Lifestyle management: Standards of Medical Care in Diabetes 2019. Diabetes Care. 2019; 42(Suppl 1):S46-S60. DOI:10.2337/dc19-S00.

67. Gillespie SJ, Kulkarni KD, Daly AE. Using carbohydrate counting in diabetes clinical practice. J Am Diet Assoc 1998; 98:897-905.

68. Vaz EC, Porfírio GJM, Nunes HRC, Nunes-Nogueira VDS. Effectiveness and safety of carbohydrate counting in the management of adult patients with type 1 diabetes mellitus: a systematic review and meta-analysis. Arch Endocrinol Metab 2018 Jun; 62(3):337-345. DOI:10.20945/2359-3997000000045.

69. Colberg S, Laan R, Dassau E, Kerr D. Physical activity and type 1 diabetes. J Diabetes Sci Technol 2015 May; 9(3): 609-618.

70. Ciccichitti A, et al. Actividad física y control glucémico, complicaciones agudas, complicaciones microvasculares y factores de riesgo cardiovascular en adultos con diabetes tipo 1. Rev ALAD 2019; 9:111-117. DOI: 10.24875/ALAD. 19000377.

71. Quirk H, Blake H, Tennyson R, RandellTL, Glazebrook C. Physical activity interventions in children and young people with type 1 diabetes mellitus: a systematic review with meta-analysis. Diabet Med 2014 Oct; 31(1):1163-1173. DOI:10.1111/dme.12531.

72. Zubizarreta-López M, Hernández-Mezquita MA, Miralles-García JM, Barrieco-Ferrero M. Tobacco and diabetes: clinical relevance and approach to smoking cessation in diabetic smokers. Endocrinología, Diabetes y Nutrición 2017; 64(4): 221-231. DOI:10.1016/j.endinu.2017.02.010.

73. Fisher L, Hessler DM, Polonsky WH, et al. Prevalence of depression in type 1 diabetes and the problem of over-diagnosis. Diabet Med 2016 Nov; 33(11):1590-1597. DOI:10.1111/dme.12973.

74. Zhu Z, Zhang F, Liu Y, et al. Relationship of obstructive sleep apnoea with diabetic retinopathy: a meta-analysis. Biomed Res Int. 2017; 2017:4737064. DOI:10.1155/2017/4737064.

75. Khouja T, Miller RG, Moore PA, Orchard TJ, Costacou T. Periodontal disease, smoking, cardiovascular complications and mortality in type 1 diabetes. Journal of Diabetes and Its Complications 2019; 33:603-609. DOI:10.1016/j.jdiacomp.2019.05.025.

76. Calixto OJ, Anaya JM. Socioeconomic status. The relationship with health and autoimmune diseases. Autoimmun Rev 2014; 13:641-54.

77. Katz M, Guo Z, Cheema A, Laffel L. Management of cardiovascular disease risk in teens with type 1 diabetes: perspectives of teens with and without dyslipemia and parents. Pediatrics Diabetes 2019; 20:210-216.

78. Giannopoulou E, Doundoulakis I, Antza C, Christoforidis A, et al. Subclinical arterial damage in children and adolescents with type 1 diabetes: a systematic review and meta-analysis. Pediatric Diabetes 2019; 20:668-677. 
79. American Diabetes Association. Children and adolescentes: Standars of Medical Care in Diabetes. Diabetes Care 2019; 42(S1):S148-S164.

80. Consenso Argentino de Hipertensión Arterial 2018. Sociedad Argentina de Hipertensión Arterial. Sociedad Argentina de Cardiología y Federación Argentina 2018. Disponible en: https:// www.sac.org.ar/wp-content/uploads/2018/08/consenso-argentino-de-hipertension-arterial-2018.pdf.

81. Elikir G, Cuneo C, Lorenzatti A, et al. Guía de práctica clínica de la Sociedad Argentina de Lípidos sobre Diagnóstico y tratamiento de las dislipemias en adultos 2019. Sociedad Argentina de Lípidos 2019. $1^{\circ}$ Edición. Disponible en: https://7be948bb6c55-43c7-b002-dd43225d11c9.filesusr.com/ugd/c4ab3f_2ce3b ebd47774974855fc7e13ee1b8f7.pdf.

82. Braffet B, Dagogo-Jack S, Bebu I, et al; DCCT/EDIC Research Group. Association of insulin dose, cardimetabolic risk factors, and cardiovascular disease in type 1 diabetes during 30 years of follow-up in the DCCT/EDIC Study. Diabetes Care 2019; 42(4):657-664.

83. Genuth S, Baclund J, Bayless M, et al. Effects of prior intensive versus conventional therapy and history of glycemia on cardiac function in type 1 diabetes in the DCC/EDIC. Diabetes 2013; 62(10):3561-3569.

84. Aviabile N, Banka A, Fonseca V. Glucose control and cardiovascular outcomes in individual with diabetes mellitus, lesson learn from the megatrials. Heart Failure Clin 8. 2012; 513-522. DOI:10.1016/j.hfc.2012.06.009.

85. What role for metformin in type 1 diabetes. Drug and Therapeutics Bulletin 2018; 56(7):78-80. DOI:10.1136/dtb.2018.7.0645.

86. Parsamanesh N, Moossavi M, Bahrami A, et al.Therapeutic potential of curcumin in diabetic complications. Pharmacological Research 2018; 136:181-193.

87. Abdelsamia E, Khaleel S, Balah A, Baky N. Curcumin augments the cardiprotective effec of meformin in an experimental model of ype I diabetes mellitus. Impact of Nrf2/HO-1 and JAK/STAT patways. Biomedicine \& Pharmacotherapy 2019; 109:21362144.

88. Doggrell S. Do glucagon-like peptide-1 receptor (GLP-1R) agonist have potential as adjuncts in the treatment of type 1 diabetes). Expert Opinion on Pharmacotherary 2018; 19:1655-1661.

89. Rovira MG, et al. Recomendaciones para el manejo de las pacientes con diabetes pregestacional. Revista de la Sociedad Argentina de Diabetes 2017; 51 (4):153-174.

90. Report of the National High Blood Pressure Education Program Working Group on High Blood Pressure in Pregnancy. Am J Obstet Gynecol 2000;183(1):S1-S22.

91. Dunne FP, Avalos G, Durkan M, et al. Atlantic Dip: pregnancy outcome for women with pregestational diabetes along the Irish Atlantic Seaboard. Diabetes Care 2009; 32: 1205-1206.

92. Xiang L, Wang Y, Lu G, Huang Q. Association of the presence of microangiopathy with adverse pregnancy outcome in type 1 diabetes: A meta-analysis. Taiwanese Journal of Obstetrics \& Gynecology 2018; 57:659-664.

93. Sacks KN, Friger M, et al. Prenatal exposure to preeclampsia as an independent risk factor for long-term cardiovascular morbidity of the offspring. Pregnancy Hypertension 2018; 13:181-186.

94. Rodríguez BL, Fujimoto WY, Mayer-Davis EJ, et al. Prevalence of cardiovascular disease risk factors in U.S. children and adolescents with diabetes: the SEARCH for Diabetes in Youth Study. Diabetes Care 2006; 29:1891-1896.

95. Schwab KO, Doerfer J, HeckerW, et al. DPV Initiative of the German Working Group for Pediatric Diabetology. Spectrum and prevalence of atherogenic risk factors in 27,358 children, adolescents, and young adults with type 1 diabetes: cross-sectional data from the German diabetes documentation and quality management system (DPV). Diabetes Care 2006; 29:218-225.

96. Margeirsdottir HD, Larsen JR, Brunborg C; Norwegian Study Group for Childhood Diabetes. High prevalence of cardiovascular risk factors in children and adolescents with type 1 diabetes: a population-based study. Diabetologia 2008; 51:554-561.
97. Robert Dalla Pozza R, Bechtold S, Bonfig W, et al. Age of onset of type 1 diabetes in children and carotid intima medial thickness. J Clin Endocrinol Metab 2007; 92: 2053-2057.

98. Urbina EM, Wadwa RP, Davis C, et al. Prevalence of increased arterial stiffness in children with type 1 diabetes mellitus differs by measurement site and sex: the SEARCH for Diabetes in Youth Study. J Pediatr 2010; 156:731-737.

99. Sepulveda-Fusaro MF, Santos Zanini JLS, Silva IN. Increased carotid intima-media thickness in Brazilian adolescents with type 1 diabetes mellitus Diabetol Metab Syndr 2016; 8:74. Published 2016 Nov 11. DOI:10.1186/s13098-016-0190-0.

100. Babar GS, Zidan $H$, et al. Impaired endothelial function in preadolescent children with type 1 diabetes. Diabetes Care 2011; 34:681-685.

101. Parikh A, Sochett EB, McCrindle BW, Dipchand A, Daneman A, Daneman D. Carotid artery distensibility and cardiac function in adolescents with type 1 diabetes. J Pediatr 2000; 137:465-469.

102. Donaghue KC, Marcovecchio ML, Wadwa RP, et al. ISPAD Clinical Practice Consensus Guidelines 2018: Microvascular and macrovascular complications in children and adolescents. Pediatric Diabetes October 2018; 19 (Suppl. 27): 262-274.

103. Guías de práctica clínica para el manejo de la diabetes mellitus tipo 1. 2011. Sociedad Argentina de Diabetes.

104. Araujo MB, Casavalle $P$, et al. Consenso sobre manejo de las dislipidemias en pediatría. Arch Argent Pediatr 2015; 113(2):177-186.

105. Marcovecchio ML, Chiesa ST, Bond S, et al. ACE inhibitors and statins in adolescents with type 1 diabetes. NEJM 2017; 377:1733-1745.

106. Marcovecchio ML, Chiesa ST, Armitage J, et al. Renal and cardiovascular risk according to tertiles of urinary albumin-tocreatinine ratio: the adolescent type 1 diabetes cardio-renal intervention trial (AdDIT). Diabetes Care 2018; 41:1963-1969.

107. Pomares $\mathrm{L}$, Braschi $\mathrm{M}$, et al. Lineamientos para la atención del consumo episódico excesivo de alcohol en adolescentes. Ministerio de Salud, Argentina. 2018. Disponible en: https:// toxicologia.org.ar/wp-content/uploads/2018/05/lineamientosatencion-alcohol-en-adolescentes1.pdf.

108. Evans-Cheung TC, Bodansky HJ, Parslow, RC. Early deaths from ischemic heart disease in childhood-onset type 1 diabetes. Archives of Disease in Childhood 2018; 103(10) 981-983.

109. Miller RG, Secrest AM, Sharma RK, Songer TJ, Orchard TJ. Improvements in the life expectancy of type 1 diabetes: the Pittsburgh Epidemiology of Diabetes Complications study cohort. Diabetes 2012; 61: 2987-2992.

110. Lee SI, Patel M, Jones CM, Narendran P. Cardiovascular disease and type 1 diabetes: prevalence, prediction and management in an ageing population. Ther Adv Chronic Disease 2015; Vol. 6(6) 347-374. DOI:10.1177/2040622315598502.

111. Chapman M, Crockett SC, Purvis T, Anderson M, Whittaker P, Bhattacharjee $\mathrm{R}$, et al. Macrovascular disease in the elderly with type 1 diabetes. J Diabetes Metab 2013; 4:8. DOI 10.4172/21556156.1000299.

112. Soedamah-Muthu S, Fuller-Mulnier HV, Raleigh VS, Lawrenson RA, Colhoun HM. All-cause mortality rates in patients with type 1 diabetes mellitus compared with a non-diabetic population from the UK general practice research database, 1992-1999. Diabetologia 2006; 49: 660-666.

113. LeRoith D, Biessels GJ, Braithwaite S, et al. Treatment of diabetes in older adults: an endocrine society clinical practice guideline. J Clin Endocrinol Metab 2019;104: 1520-1574.

114. Sinclair AJ, Dunning T, Dhatariya K; an International Group of Experts. Clinical guidelines for type 1 diabetes mellitus with an emphasis on older adults: an Executive Summary. Diabet Med 2020; 37(1):53-70. DOI:10.1111/dme.14135.

115. Joint British Societies consensus recommendations for the prevention of cardiovascular disease (JBS3). Heart 2014;100:ii1-ii67.

116. Keenan $\mathrm{H}$, Sun JK, Levine J, et al. Residual insulin production and pancreatic cell turnover after 50 years of diabetes: Joslin Medalist Study. Diabetes 2010; 59:2846-2853. DOI:10.2337/db10-0676. 
Revista de la Sociedad Argentina de Diabetes Vol. 54 № 2 Suplemento I Jornadas Conjuntas del Comité de Graduados y el Comité de Hipertensión Arterial y otros FRCV. XVIII Jornadas del Comité de Graduados

Mayo-agosto de 2020: 71-90 ISSN 0325-5247 (impresa) ISSN 2346-9420 (en línea)

117. Bain SC, Gill GV, Dyer PH, et al. Characteristics of type 1 diabetes of over 50 years duration (the Golden Years Cohort). Diabetic Medicine 2003; 20:808-811. DOI:10.1046/j.14645491.2003.01029.x.

118. Lindahl JP, Hartmann A, Horneland R, et al. Improved patient survival with simultaneous pancreas and kidney transplantation in recipients with diabetic end-stage renal disease. Diabetologia 2013; 56:1364-1371. DOI:10.1007/s00125-013-2888-y.

119. Luan F, Miles C, Cibrik D, Ojo A. Impact of simultaneous pancreas and kidney transplantation on cardiovascular risk factors in patients with type 1 diabetes mellitus. Transplantation Aug 2007; Vol 84 (4):541-544. DOI:10.1097/01.tp.0000270617.43811.af.

120. Van Dellen D, Worthington J, Mitu-Pretorian OM, et al. Mortality in diabetes: Pancreas transplantation is associated with significant survival benefit. Nephrol Dial Transplant 2013; 28:1315-22. DOI:10.1093/ndt/gfs613.

121. J.M. Maroto Montero Unidad de Rehabilitación Cardíaca. Servicio de Cardiología. Hospital Ramón y Cajal. Madrid. ISBN: 978-84-88336-74-3

122. Piepoli MF, Corra U, Adamopoulos S, et al. Secondary prevention in the clinical management of patients with cardiovascular diseases. Core components, standards and outcome measures for referral and delivery: a policy statement from the cardiac rehabilitation section of the European Association for Cardiovascular Prevention \& Rehabilitation. Endorsed by the Committee for Practice Guidelines of the European Society of Cardiology. Eur J Prev Cardiol 2014; 21:664-681.
123. Piepoli MF, Hoes AW, Agewall S, et al. 2016 European guidelines on cardiovascular disease prevention in clinical practice: The Sixth Joint Task Force of the European Society of Cardiology and Other Societies on Cardiovascular Disease Prevention in Clinical Practice. Eur Heart J 2016; 37:2315-2381.

124. Suaya JA, Stason WB, Ades PA, et al. Cardiac rehabilitation and survival in older coronary patients. J Am Coll Cardiol 2009; 54:25-33.

125. Anderson L, Oldridge N,Thompson DR, et al. Exercise-based cardiac rehabilitation for coronary heart disease: Cochrane Systematic Review and Meta-Analysis. J Am Coll Cardiol 2016;67:1-12.

126. Lear SA, HuW, Rangarajan S, et al. The effect of physical activity on mortality and cardiovascular disease in 130000 people from 17 high-income, middle-income, and low-income countries: the PURE study. Lancet 2017; 390:2643-2654.

127. Chatziefstratiou A, Fotos NV, et al. The contribution of cardiac rehabilitation program in management of diabetes mellitus: $\mathrm{A}$ systematic review. Obesity Medicine 2019; 15. DOI:10.1016/j.obmed.2019.100103. 